Article

\title{
Synthesis of 1,2,3-Triazolium Ionic Liquid-Supported Chiral Imidazolidinones and Their Application in Asymmetric Alkylation Reaction
}

\author{
Yunkyung Jeong, Yunjeong Park and Jae-Sang Ryu *(i) \\ College of Pharmacy \& Graduate School of Pharmaceutical Sciences, Ewha Womans University, \\ 52 Ewhayeodae-gil, Seodaemun-Gu, Seoul 03760, Korea; echo4001@naver.com (Y.J.); \\ amethyst-21@hanmail.net (Y.P.) \\ * Correspondence: ryuj@ewha.ac.kr; Tel.: +82-2-3277-3008; Fax: +82-2-3277-2851 \\ Academic Editor: Wim Dehaen \\ Received: 19 August 2019; Accepted: 13 September 2019; Published: 14 September 2019
}

\begin{abstract}
New 1,2,3-triazolium ionic liquid-supported chiral imidazolidinones were developed. The feasibility of the ionic liquid-supported imidazolidinones as chiral auxiliaries was demonstrated in sequential propionylation-alkylation-cleavage reactions, which provided the chiral product with good to excellent chemical yields (up to $90 \%$ ) and high selectivities (up to $94 \%$ ee). The progress of the reactions could be monitored by TLC and NMR, and the ionic liquid-supported chiral auxiliaries could be recovered by simple extraction.
\end{abstract}

Keywords: ionic liquid; 1,2,3-triazolium; chiral imidazolidinone; asymmetric alkylation; chiral auxiliaries

\section{Introduction}

Since the Evans' group first developed enantioselective alkylation reactions using chiral oxazolidinone enolates in 1980 [1], oxazolidinone auxiliaries have been extensively studied and widely applied to many asymmetric reactions, such as aldol [2,3], alkylation [4,5], Diels-alder [6], conjugated addition [7], and other reactions. As shown over the course of several decades, the use of chiral oxazolidinones offers several benefits in asymmetric reactions: Easy preparation, easy installation, easy removal, high selectivity, and recyclability. However, Evans' chiral auxiliaries are expensive and recovering them is tedious work. Due to these issues, continued efforts have been made over the past decades to develop polymer-supported forms [8] of Evans' chiral auxiliaries as convenient and practical tools for producing chiral compound libraries. The major advantages of this method are rapid isolation of the desired chiral product and facile recovery of the polymer-supported auxiliary by simple filtration. Nevertheless, their use in asymmetric reactions has been problematic [9-12]. Indeed, a relatively narrow range of reaction conditions and substrates have been covered, and inconsistent yields and stereoselectivity levels have been observed compared to the analogous solution-phase reactions [9-12]. These problems can be ascribed to the presence of polymer supports that are insoluble in the reaction media $[13,14]$. In general, the heterogeneous reaction conditions of solid-phase synthesis cause several issues, including site-site interactions [15], nonlinear kinetic behavior, diffusion-limited reactivity, unequal distribution of reagents, reduced reactivity, extended reaction times, and reagent leaching. Owing to these drawbacks, soluble matrices such as poly(ethylene glycol) [16-18], linear polystyrenes [19], and polyethylenes [20] have drawn increasing attention as alternative supports in combinatorial synthesis [21]. However, soluble polymer-supported synthesis can be wasteful because a large amount of solvents is required to precipitate the polymer support at the end of the reaction. While many attempts to develop efficient matrices for chiral auxiliaries have been made, the results have been 
unsatisfactory. Currently, it is highly desirable and necessary to develop new soluble matrix-supported reusable chiral auxiliaries.

In contrast to insoluble polymers, ionic liquid-supported compounds are soluble in certain organic solvents, and are compatible with most common reagents [22]. In particular, the recovery of ionic liquid-supported material is easily accomplished by simple extraction after a reaction is complete [23]. In this context, ionic liquids have been considered as alternative soluble supports for Evans' chiral auxiliaries. Among several potential ionic liquids, 1,2,3-triazolium ionic liquid was selected as the best ionic liquid support. Previously, we reported the use of 1,3-dialkyl-1,2,3-triazolium ionic liquids as stable and recyclable solvents [24]. The applicability of these ionic liquids as new reaction media was also shown for the Baylis-Hillman reaction, where 1,3-dialkyl-1,2,3-triazolium ionic liquids exhibited properties comparable to those of imidazolium ionic liquids in terms of thermal stability and miscibility. Compared with imidazolium ionic liquids, 1,2,3-triazolium ionic liquids are chemically inert under acidic [25] or basic [24] conditions, which make them more suitable media or supports for acid- or base-mediated reactions. Therefore, 1,2,3-triazolium ionic liquids are considered to be the best supports for Evans' chiral auxiliaries. Herein, we first reports ionic liquid-supported Evans' chiral auxiliaries (Figure 1). Compared to solid-supported chiral auxiliaries, 1,2,3-triazolium ionic liquid-supported chiral auxiliaries possess several advantages: i) The reaction can be conducted under homogeneous conditions; ii) it is easy to monitor progress of the reaction using TLC; iii) it is not necessary to translate solution-phase reactions to solid-phase reactions because there is no insoluble polymer support that can have a significant effect on both reactivity and selectivity; iv) ionic liquid-supported intermediates/products can be monitored by NMR after removal of the solvents; $v$ ) the product can easily be isolated and ionic liquid-supported chiral auxiliaries can be recycled by simple extraction.
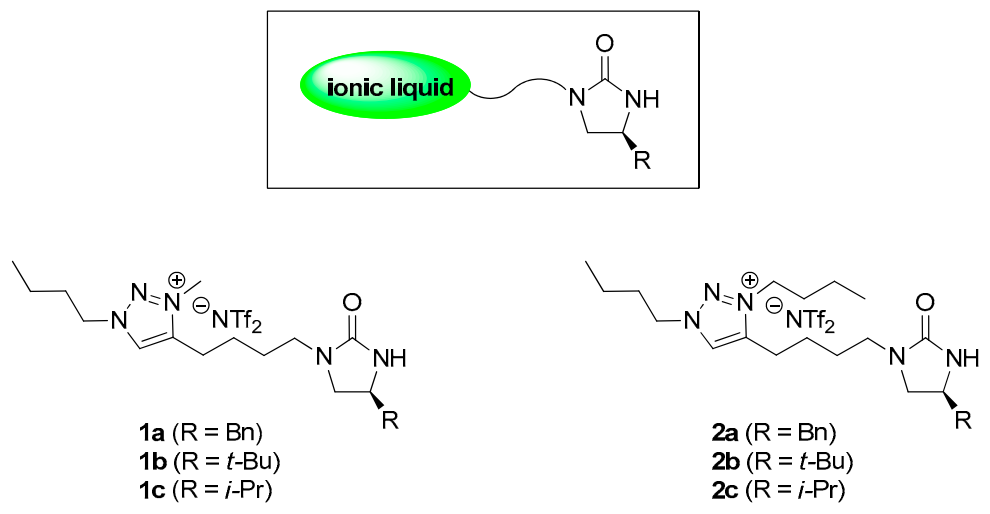

Figure 1. 1,2,3-Triazolium ionic liquid-supported chiral imidazolidinone auxiliaries $\mathbf{1 a - c}$ and $\mathbf{2 a - c}$.

\section{Results and Discussion}

\subsection{Synthesis of Ionic Liquid-supported Imidazolidinone $\mathbf{1 a - c}$}

1,2,3-Triazolium-tethered imidazolidinone $\mathbf{1 a}-\mathbf{c}$ and $\mathbf{2 a}-\mathbf{c}$ were easily produced by $\mathrm{Cu}$-catalyzed Huisgen 1,3-dipolar cycloaddition [26] using $n$-butyl azide and alkyne (Scheme 1), which proved useful for the synthesis of substituted 1,2,3-triazolium ionic liquids. Synthesis commenced with the azidation of commercially available $n$-butyl bromide (3). In situ generated $n$-butyl azide (4) was captured by $N$-(5-hexynyl)phthalimide via a Cu-catalyzed click reaction, which gave 1,4-disubstituted 1,2,3-triazole 5 at an $89 \%$ yield in two steps. The phthalimide moiety was then ruptured by aminolysis using hydrazine-hydrate to afford a primary amine 6 at a yield of $98 \%$, which was readily condensed with Boc-protected amino acids in the presence of EDCI.HCl and HOBt. In this step, three amino acids carrying benzyl, $t$-butyl, and $i$-propyl substituents were coupled to 6 to synthesize benzyl-, $t$-butyl-, and $i$-propyl-substituted imidazolidinones. The subsequent treatment of the Boc-protected amines $7 \mathbf{a}-\mathbf{c}$ with TFA led to the production of the primary amines $8 \mathbf{a}-\mathbf{c}$ in almost quantitative yields. The 
amide moieties of $\mathbf{8 a}-\mathbf{c}$ were reduced with $\mathrm{LiAlH}_{4}$ to yield the diamines $\mathbf{9 a}-\mathbf{c}$, which were converted into the imidazolidinones $10 \mathrm{a}-\mathrm{c}$ using $\mathrm{CDI}$ and $\mathrm{Et}_{3} \mathrm{~N}$.
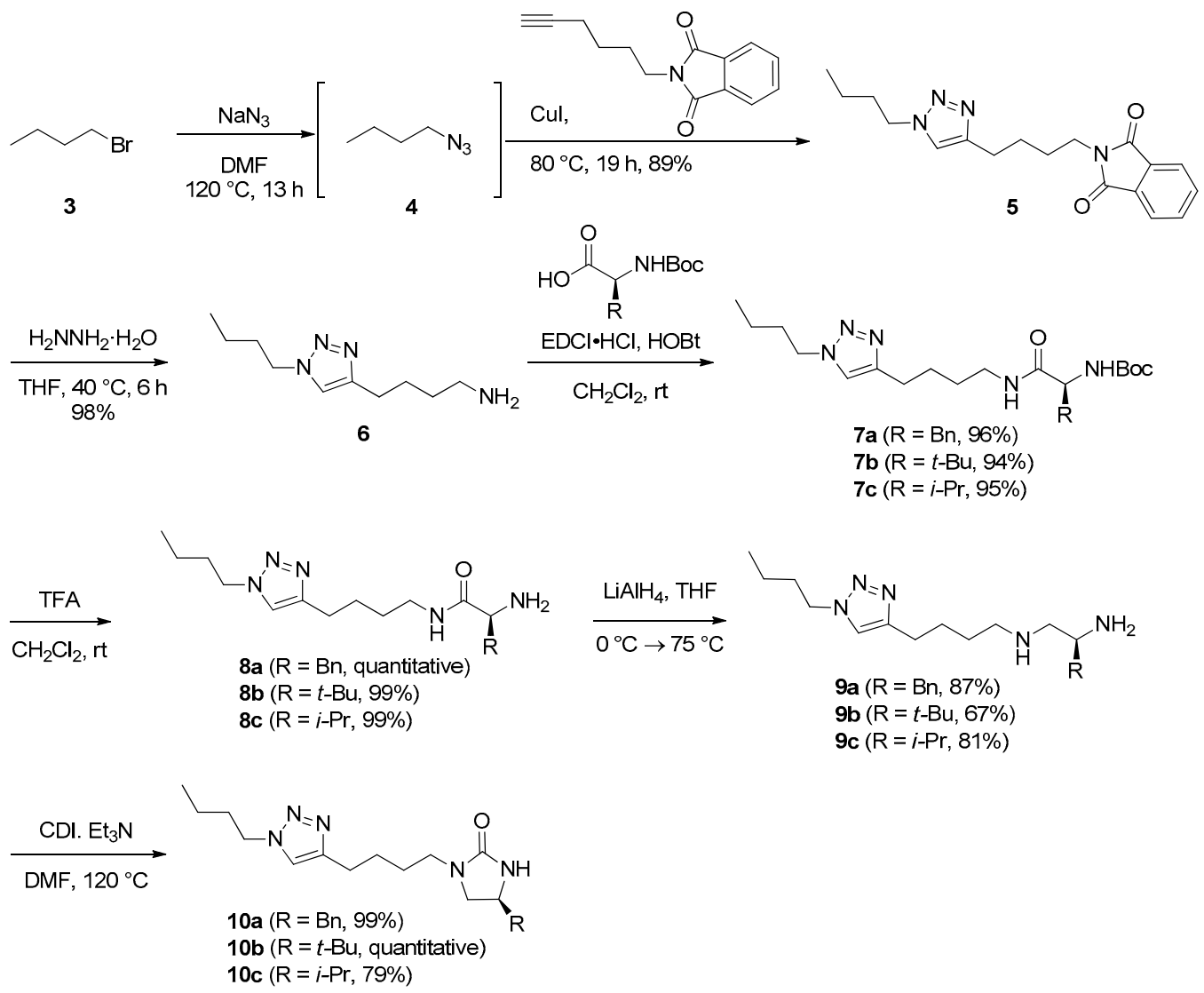

Scheme 1. Synthesis of (S)-4-alkyl-1-(4-(1-butyl-1H-1,2,3-triazol-4-yl)butyl)imidazolidin-2-ones 10a-c.

At this stage, we needed to confirm that no epimerization occurred throughout the synthesis route shown in Scheme 1. Therefore, the enantiopurity of the synthesized 1,2,3-triazole-tethered (S)-4-benzyl-2-imidazolidinone 10a was examined by Mosher's method [27,28]. For this purpose, 1,2,3-triazole-tethered $(R)$-4-benzyl-2-imidazolidinone $\mathbf{1 0 \mathbf { a } ^ { \prime }}$ was also prepared by the same synthetic route using Boc-D-Phe-OH instead of Boc-L-Phe-OH (Scheme S1, Supplementary Materials). With both 1,2,3-triazole-tethered (S)-4-benzyl-2-imidazolidinone 10a and (R)-4-benzyl-2-imidazolidinone $\mathbf{1 0}^{\prime}$ in hand, the enantiopurity was determined by NMR. Synthesized 1,2,3-triazole-tethered (S)-4-benzyl-2-imidazolidinone 10a and (R)-4-benzyl-2-imidazolidinone 10 $\mathbf{a}^{\prime}$ were separately treated with $(R)$-Mosher's acid chloride to obtain crude Mosher's imide 11a and 11a' , respectively, as shown in Scheme 2. After analyzing the NMR spectra of the crude mixtures, it was confirmed that both 10a and $\mathbf{1 0 \mathbf { a } ^ { \prime }}$ were enantiomerically pure, and no epimerization occurred during synthesis. 


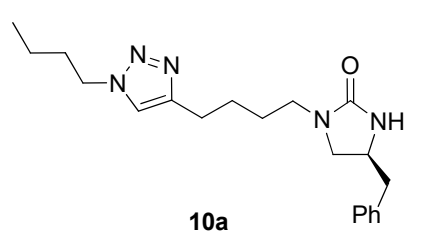

$10 a$

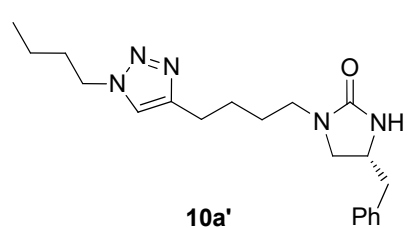

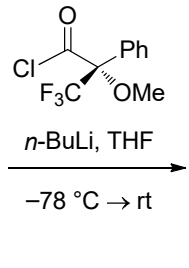

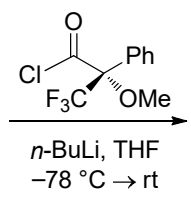

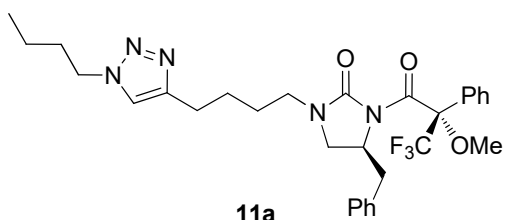

$11 \mathrm{a}$

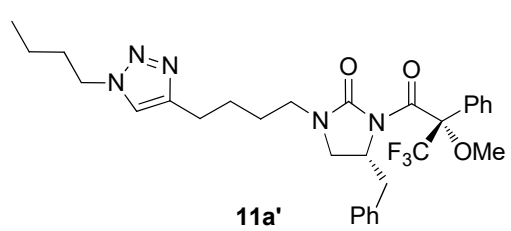

Scheme 2. Synthesis of (S)-4-benzyl-1-[4-(1-butyl-1H-1,2,3-triazol-4-yl)butyl)-3-((S)-2-methoxy-2trifluoromethylphenylacetyl)imidazolidin-2-one (11a) and (R)-4-benzyl-1-[4-(1-butyl-1H-1,2,3-triazol-4yl)butyl]-3-((S)-2-methoxy-2-trifluoromethylphenylacetyl)imidazolidin-2-one (11a').

The 1-butyl-3-methyl-1,2,3-triazolium ionic liquid-supported imidazolidinones 1a-c were synthesized according to Scheme 3. The 1-butyl-1,2,3-triazole-tethered imidazolidinones 10a-c were methylated with methyl iodide under neat conditions, which afforded 1-butyl-3-methyl-1,2,3-triazolium iodide salts 12a-c in the form of sticky oils. Then, the 1-butyl-3-methyl-1,2,3-triazolium iodide salts 12a-c were treated with $\mathrm{LiNTf}_{2}$ in deionized water to produce 1,2,3-triazolium NTf $\mathrm{Nalts}_{2}$ 1a-c.

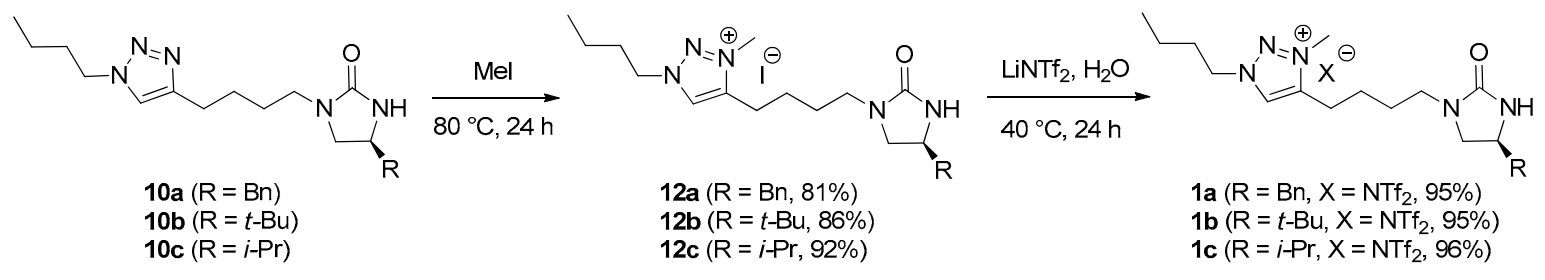

Scheme 3. Synthesis of the 1-butyl-3-methyl-1,2,3-triazolium ionic liquid-supported imidazolidinones 1a-c.

All synthesized 1-butyl-3-methyl-1,2,3-triazolium ionic liquid-supported imidazolidinones 12a-c and $\mathbf{1 a}-\mathbf{c}$ were liquids at room temperature and considered room temperature ionic liquids (RTILs) [29]. Especially, the 1,2,3-triazolium $\mathrm{NTf}_{2}$ salts $\mathbf{1 a}$-c were liquids even at $-78^{\circ} \mathrm{C}$. The 1,2,3-triazolium $\mathrm{NTf}_{2}$ salts 1a-c dissolved well in THF at $-78{ }^{\circ} \mathrm{C}$. Even though $n$-BuLi was added in the THF solution at $-78{ }^{\circ} \mathrm{C}$, it remained as a clear solution. Thus, the $\mathrm{NTf}_{2}$ salts $\mathbf{1 a}-\mathbf{c}$ were considered useful for low-temperature asymmetric reactions.

\subsection{Application to Asymmetric Reactions}

To assess the feasibility of asymmetric alkylation reactions using the ionic liquid-supported chiral auxiliaries 1a-c, sequential propionylation-alkylation-cleavage reactions beginning with $1 \mathbf{a}-\mathbf{c}$ (Scheme 4) began to be investigated. Propionylation of $\mathbf{1 a}-\mathbf{c}$ was performed using propionyl chloride and $n$ - $\mathrm{BuLi}$ as a base at $-78{ }^{\circ} \mathrm{C}$, and yielded 13a-c. All reagents were soluble in THF at $-78{ }^{\circ} \mathrm{C}$ and the reaction mixture remained homogeneous throughout the reaction. The progress of the reactions was monitored by TLC and the formation of $13 a-c$ was successfully verified by ${ }^{1} \mathrm{H}-\mathrm{NMR}$ of the crude mixture after completion of the reaction. Although $13 \mathbf{a}-\mathbf{c}$ could be purified by column chromatography, this study demonstrated the feasibility of the ionic liquids as liquid supports for Evans' auxiliaries without column chromatography. Therefore, after the propionylation reaction, asymmetric alkylation reactions of 13a-c using 3-chlorobenzyl bromide and NaHMDS followed the propionylation immediately after the separation of the crude products $13 \mathbf{a}-\mathbf{c}$ from excess reagents by simple extraction. Then, the resulting crude alkylated products $14 \mathbf{a}-\mathbf{c}$ were treated with aqueous 2 
$N \mathrm{NaOH}$ solution in 1,4-dioxane at room temperature to cleave chiral products, which gave chiral (-)-3-(3-chlorophenyl)-2-methylpropionic acid (15) and regenerated the crude 1,2,3-triazolium $\mathrm{NTf}_{2}$ salts 1a-c. Upon completion of the reaction, the product was separated by simple extraction with EtOAc after acidification with $10 \% \mathrm{HCl}$. The isolated $\mathbf{1 5}$ was purified by column chromatography and the enantiomeric excess (ee) was determined by chiral HPLC after modification to the corresponding $\mathrm{N}$-phenylpropanamide.

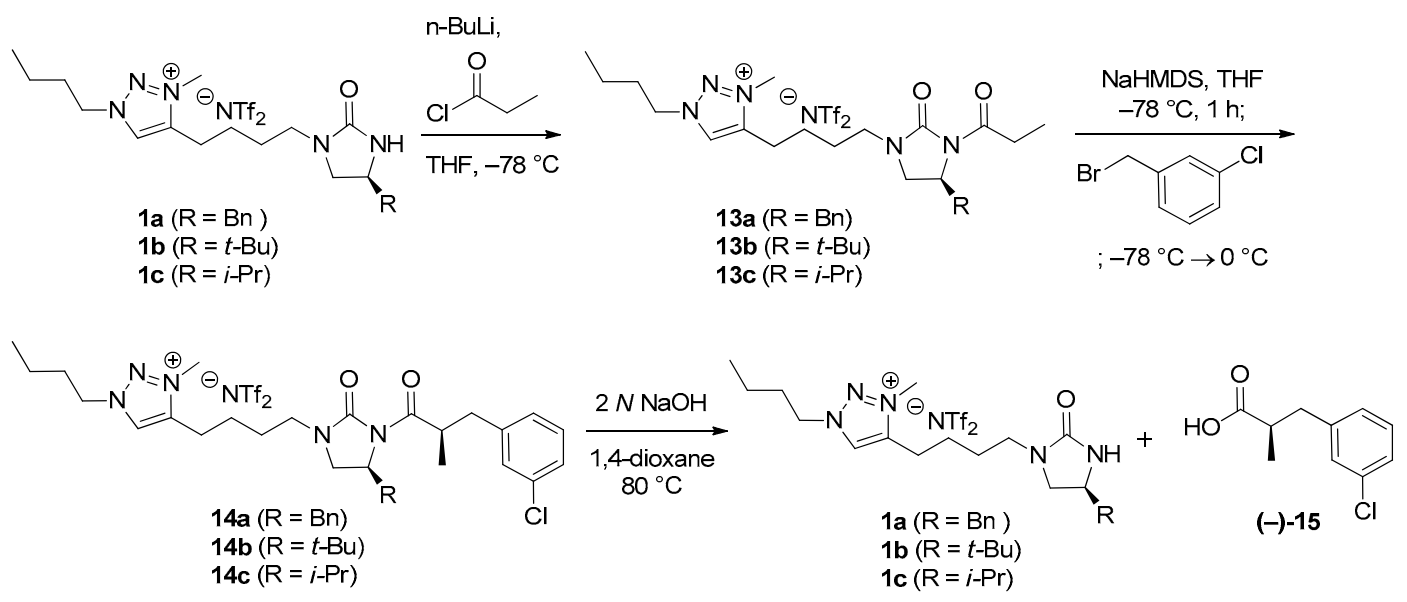

Scheme 4. Asymmetric synthesis of (R)-(-)-3-(3-chlorophenyl)-2-methylpropionic acid (15) using 1a-c.

Table 1 shows the yields and ee's of the sequential three-step reactions using the 1-butyl-3-methyl-1,2,3-triazolium ionic liquid-supported imidazolidinones 1a-c, which functioned as efficient chiral auxiliaries in terms of selectivity. In the sequential reactions using $\mathbf{1 a}-\mathbf{c}$, the ee values were as high as $91-93 \%$, but moderate yields were observed. Therefore, each step of the sequential reactions was scrutinized. Interestingly, an $\mathrm{N}$-methylated byproduct $\mathbf{1 3 \mathbf { c } ^ { \prime }}$ was isolated in the propionylation reaction using 1c after column chromatography (Scheme 5), the structure of which was unambiguously identified as an $\mathrm{N}$-methylated imidazolidinone-ionic liquid 13c' by 2D NMR experiments (NOESY, COSY, and HMBC, Supplementary Materials). Possibly, when propionylation of $\mathbf{1 a}-\mathbf{c}$ was performed using $n$-BuLi solution as a base at $-78{ }^{\circ} \mathrm{C}$, the anion generated in situ by deprotonation of imidazolidinone abstracted the methyl group from the 1-butyl-3-methyl-1,2,3-triazolium cation intermolecularly. It was found that the methyl group of 1-butyl-3-methyl-1,2,3-triazolium could act as a methylating agent. Therefore, the ionic liquids were modified to improve stability.

Table 1. Yield and enantiomeric excess (ee) of (-)-15 in ionic liquid (1a-c)-supported reactions. ${ }^{a}$

\begin{tabular}{cccc}
\hline Entry & IL-Supported Imidazolidinone & Yield (\%) $^{\boldsymbol{a}}$ & ee $^{\boldsymbol{b}}$ \\
\hline 1 & 1a & 66 & 91 \\
2 & $\mathbf{1 b}$ & 49 & 93 \\
3 & 1c & 59 & 93 \\
\hline
\end{tabular}

${ }^{a}$ Isolated yields in three steps. ${ }^{b}$ Determined by HPLC analysis of the corresponding $N$-phenylpropanamide on a chiral stationary phase.
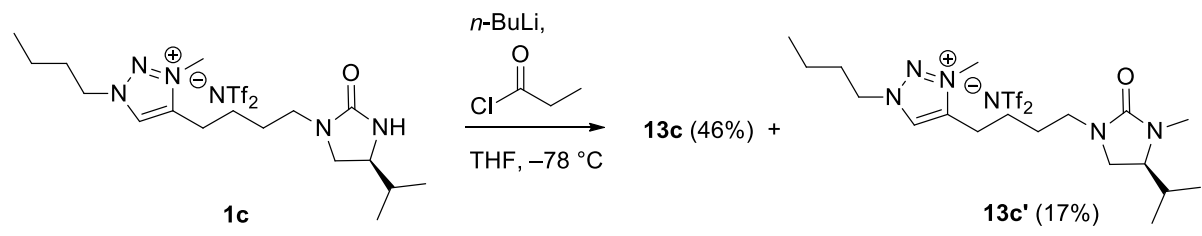

Scheme 5. The formation of $N$-methylated byproduct $13 c^{\prime}$ in the propionylation reaction using $1 \mathrm{c}$. 


\subsection{Synthesis of Ionic Liquid-Supported Imidazolidinone $\mathbf{2 a - c}$}

As the methyl group of 1-butyl-3-methyl-1,2,3-triazolium-supported isopropyl imidazolidinone 1c was turned out labile under the propionylation reaction conditions, it was decided to incorporate a more sterically hindered $n$-butyl group into the 1,2,3-triazolium core instead of the methyl group in order to develop more versatile supports and to avoid undesirable side reactions. Based on

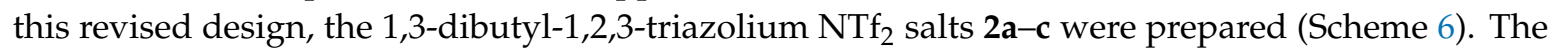
disubstituted triazoles 10a-c were butylated using $n$-butyl iodide under neat conditions, which afforded 1,3-dibutyl-1,2,3-triazolium iodide salts $16 \mathbf{a}-\mathbf{c}$ in $77-92 \%$ yields. Then, the iodide salts $16 \mathbf{a}-\mathbf{c}$ were treated with $\mathrm{LiNTf}_{2}$ in deionized water for anion metathesis, which produced the 1,2,3-triazolium $\mathrm{NTf}_{2}$ salts 2a-c. All synthesized 1,3-dibutyl-1,2,3-triazolium-supported imidazolidinones 16a-c and $\mathbf{2 a}-\mathbf{c}$ were liquids at room temperature and were considered room temperature ionic liquids (RTILs). The 1,2,3-triazolium $\mathrm{NTf}_{2}$ salts $\mathbf{2 a} \mathbf{a}-\mathbf{c}$ were liquid even at $-78{ }^{\circ} \mathrm{C}$ and were suitable for low-temperature asymmetric reactions.

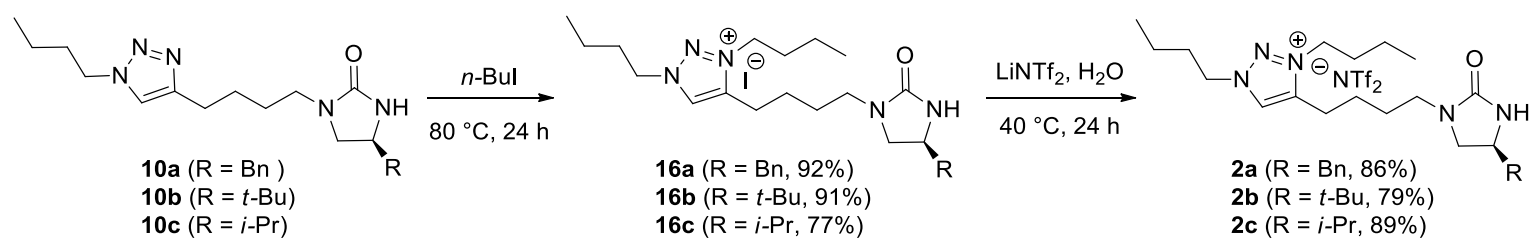

Scheme 6. Synthesis of the 1,3-dibutyl-1,2,3-triazolium ionic liquid-supported imidazolidinones 2a-c.

\subsection{Application to Asymmetric Reactions}

With the more sterically hindered 1,3-dibutyl-1,2,3-triazolium-supported imidazolidinones 2a-c in hand, the same feasibility tests as those done for ionic liquid-supported chiral auxiliaries were performed (Scheme 7). Sequential propionylation- alkylation-cleavage reactions were carried out as described above, and (-)-3-(3-chlorophenyl)-2-methylpropionic acid (15) was obtained. As shown in Table 2, high yields and ee's were observed for all reactions using the 1,3-dibutyl-1,2,3-triazolium $\mathrm{NTf}_{2}$ salts $\mathbf{2 a - c}$. The crude product (-)-15 obtained after cleavage was very pure, as shown in Figure 2 . Notably, no disruption of either the imidazolidinone ring or the 1,2,3-triazolium was observed for all recovered $\mathbf{2 a}-\mathbf{c}$ after cleavage with $2 \mathrm{~N} \mathrm{NaOH}[30,31]$. In particular, the corresponding $\mathrm{N}$-methylated byproducts were not observed and most of the recovered ionic liquid-supported chiral auxiliaries remained intact. As the reactions were conducted under homogeneous conditions, it was not necessary to translate solution-phase reactions to solid-phase reactions. Thus, the progress of the reactions could be monitored by TLC and NMR, which revealed consistent yield and selectivity. The inconsistent yield and selectivity problems that originated from heterogeneous solid-phase reactions were resolved by the use of ionic liquid-supported imidazolidinones.

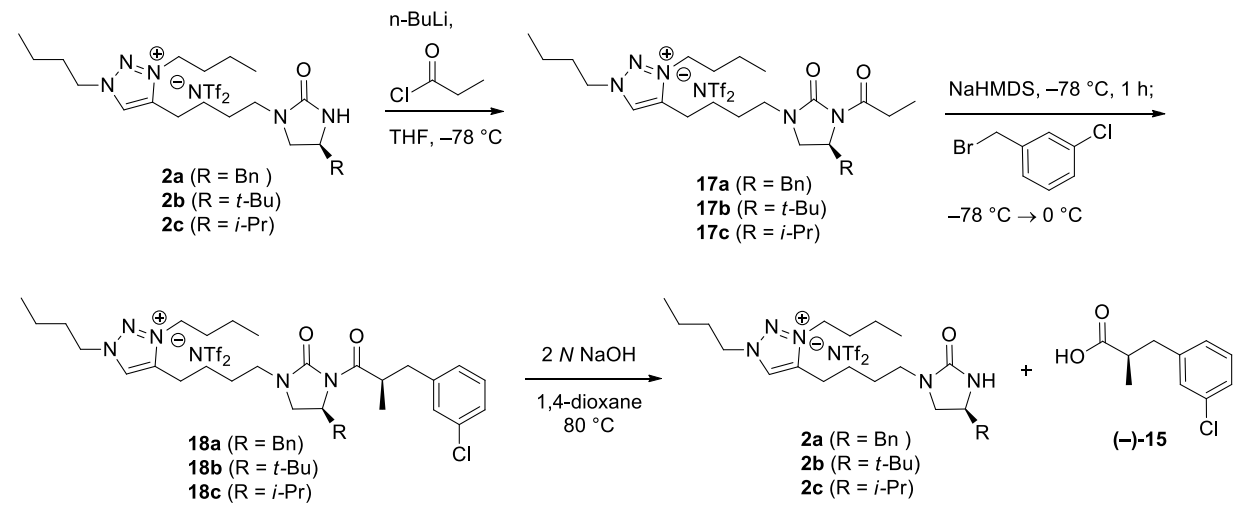

Scheme 7. Asymmetric synthesis of (R)-(-)-3-(3-chlorophenyl)-2-methylpropionic acid (15) using 2a-c. 
Table 2. Yields and enantiomeric excess (ee) of (-)-15 in ionic liquid (2a-c)-supported reactions. ${ }^{a}$

\begin{tabular}{cccc}
\hline Entry & IL-Supported Imidazolidinone & Yield (\%) $^{\boldsymbol{a}}$ for 3 Steps & ee $^{\boldsymbol{b}}$ \\
\hline 1 & $\mathbf{2 a}$ & 90 & 90 \\
2 & $\mathbf{2 b}$ & 81 & 90 \\
3 & $\mathbf{2 c}$ & 80 & 94 \\
\hline
\end{tabular}

$\bar{a}$ Isolated yields in three steps. ${ }^{b}$ Determined by HPLC analysis of the corresponding $N$-phenylpropanamide on a chiral stationary phase.

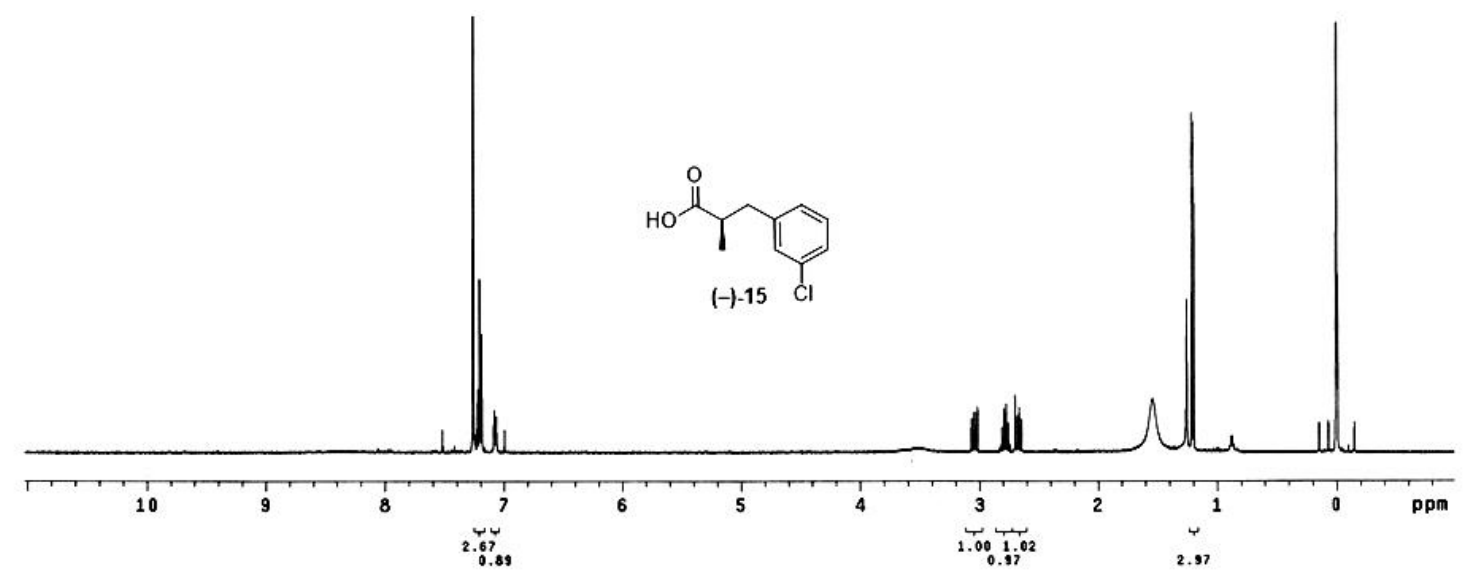

Figure 2. The NMR spectrum of crude (-)-15 obtained after cleavage in the sequential reactions using $\mathbf{2 a}$.

It is particularly interesting whether the presence of the ionic liquid-tether lowers the ee or not. Thus, the enantiomeric excesses of the reactions using 2a with those using Evans' auxiliary (Scheme 8) were directly compared. The reactions were conducted under the standard conditions used in the Scheme 7, and observed 91\% ee for (-)-15. As expected, the obtained ee's for both reactions were comparable (91\% ee for Scheme 8 versus $90 \%$ ee for Table 2, entry 1 ), and no decrease of the ee's was observed. The 1,2,3-triazolium ionic liquid-tether did not affect the stereoselectivity.

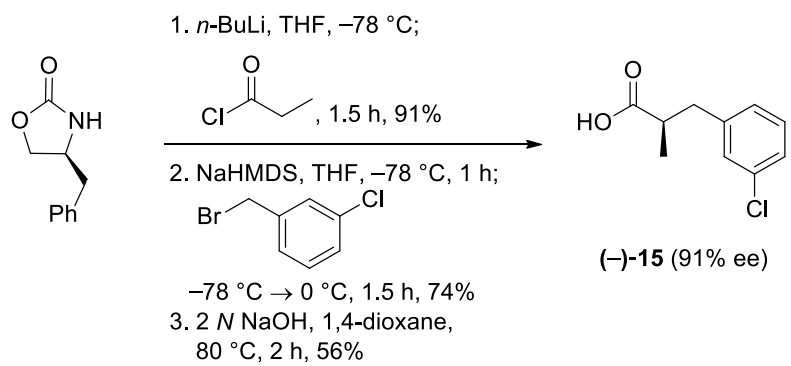

Scheme 8. Asymmetric synthesis of (R)-(-)-3-(3-chlorophenyl)-2-methylpropionic acid (15) using Evans' auxiliary.

\section{Materials and Methods}

\subsection{General Methods}

All reactions were performed in oven-dried glassware fitted with a glass stopper under positive pressure of Ar with magnetic stirring, unless otherwise noted. The air- and moisture-sensitive liquids and solutions were transferred via syringe or stainless-steel cannula. TLC was performed on $0.25 \mathrm{~mm}$ E. Merck silica gel 60 F254 plates (Merck, Kenilworth, IL, USA) and visualized under UV light (254 nm) or by staining with cerium ammonium molybdenate (CAM), potassium permanganate $\left(\mathrm{KMnO}_{4}\right)$ or p-anisaldehyde. Flash chromatography was performed on E. Merck 230-400 mesh silica gel 60 (Merck). The reagents were purchased from commercial suppliers, and used without further purification unless 
otherwise noted. The solvents were distilled from proper drying agents $\left(\mathrm{CaH}_{2}\right.$ or Na wire) under Ar atmosphere at $760 \mathrm{~mm} \mathrm{Hg}$. All moisture- and/or oxygen-sensitive solids were handled and stored in a glove box under $\mathrm{N}_{2}$. The NMR spectra were recorded on at $24{ }^{\circ} \mathrm{C}$. The chemical shifts were expressed in ppm relative to TMS $\left({ }^{1} \mathrm{H}, 0 \mathrm{ppm}\right), \mathrm{CDCl}_{3}\left({ }^{1} \mathrm{H}, 7.26 \mathrm{ppm} ;{ }^{13} \mathrm{C}, 77.2 \mathrm{ppm}\right), \mathrm{DMSO}-d_{6}\left({ }^{1} \mathrm{H}, 2.50 \mathrm{ppm}\right.$; $\left.{ }^{13} \mathrm{C}, 39.5 \mathrm{ppm}\right)$, acetone- $d_{6}\left({ }^{1} \mathrm{H}, 2.05 \mathrm{ppm} ;{ }^{13} \mathrm{C}, 206.3,29.9 \mathrm{ppm}\right), \mathrm{CD}_{3} \mathrm{OD}\left({ }^{1} \mathrm{H}, 3.31 \mathrm{ppm} ;{ }^{13} \mathrm{C}, 49.1 \mathrm{ppm}\right)$ or $\mathrm{C}_{6} \mathrm{H}_{5} \mathrm{~F}\left({ }^{19} \mathrm{~F},-113.15 \mathrm{ppm}\right)$; coupling constants were expressed in $\mathrm{Hz}$. The high resolution mass spectra (HRMS) were obtained by ESI (TOF analyzer) or FAB (magnetic sector analyzer). The optical rotations were determined in acetone, $\mathrm{CHCl}_{3}$ or $\mathrm{MeOH}$. Chiral HPLC analysis for the determination of the enantomeric excess (ee) was performed on a chiral Lux $5 u$ Cellulose- 1 column $(5 \mu \mathrm{m}, 1000 \AA$, $250 \times 4.6 \mathrm{~mm}$ ). The solvents were eluted at a flow rate of $0.8 \mathrm{~mL} / \mathrm{min}$ at room temperature using a binary solvent system (solvent A: hexane, solvent B: isopropanol, $90 \%$ A over $30 \mathrm{~min}$ ) with UV detection at $254 \mathrm{~nm}$.

\subsection{Synthesis}

N-[4-(1-Butyl-1H-1,2,3-triazol-4-yl)butyl]phthalimide (5). In a screw-cap vial, a mixture of 1-bromobutane (3) $(800 \mu \mathrm{L}, 7.29 \mathrm{mmol})$ and sodium azide $(949 \mathrm{mg}, 14.6 \mathrm{mmol})$ in anhydrous DMF (11 mL) was stirred at $120^{\circ} \mathrm{C}$ for $14 \mathrm{~h}$. After the mixture was cooled to rt, $\mathrm{N}$-(5-hexynyl)phthalimide $(1.66 \mathrm{~g}, 7.29 \mathrm{mmol})$ and $\mathrm{CuI}(139 \mathrm{mg}, 730 \mu \mathrm{mol})$ were added, and the solution was stirred at $80{ }^{\circ} \mathrm{C}$ for $19 \mathrm{~h}$. Upon completion of the reaction, the reaction mixture was cooled to $\mathrm{rt}$ and concentrated in vacuo. The purification by column chromatography on silica gel (3:1 hexanes/EtOAc) afforded a desired triazole $5(2.13 \mathrm{~g}, 89 \%)$ as a light-yellow solid. TLC: Rf 0.21 (3:1 hexanes/EtOAc). mp: $73.4-74.8^{\circ} \mathrm{C} .1 \mathrm{H} \mathrm{NMR}(400 \mathrm{MHz}, \mathrm{CDCl} 3)$ : $\delta 7.84(\mathrm{dd}, \mathrm{J}=5.6,2.8 \mathrm{~Hz}, 2 \mathrm{H}), 7.71(\mathrm{dd}, \mathrm{J}=5.6,2.8 \mathrm{~Hz}, 2 \mathrm{H}), 7.28(\mathrm{~s}, 1 \mathrm{H}), 4.31(\mathrm{t}, \mathrm{J}=7.2 \mathrm{~Hz}, 2 \mathrm{H}), 3.72(\mathrm{t}$, $\mathrm{J}=6.8 \mathrm{~Hz}, 2 \mathrm{H}), 2.77(\mathrm{t}, \mathrm{J}=6.8 \mathrm{~Hz}, 2 \mathrm{H}), 1.87$ (quintet, $\mathrm{J}=7.2 \mathrm{~Hz}, 2 \mathrm{H}), 1.77-1.71(\mathrm{~m}, 4 \mathrm{H}), 1.35$ (sextet, $\mathrm{J}=7.2 \mathrm{~Hz}, 2 \mathrm{H}), 0.95$ (t, J = 7.2 Hz, 3H). 13C NMR (100 MHz, CDCl3): $\delta$ 168.6, 147.6, 134.1, 132.3, 123.3, $120.7,50.0,37.8,32.5,28.3,26.9,25.3,19.9,13.6$. LRMS (ESI) $m / z$ (rel int): (pos) $327\left([\mathrm{M}+\mathrm{H}]^{+}, 100\right), 282$ $\left(\left[\mathrm{M}-\mathrm{C}_{3} \mathrm{H}_{8}\right]^{+}, 100\right)$. HRMS (ESI) $\mathrm{m} / \mathrm{z}$ calcd for $\mathrm{C} 18 \mathrm{H} 23 \mathrm{~N} 4 \mathrm{O} 2^{+}\left([\mathrm{M}+\mathrm{H}]^{+}\right)$327.1816, found 327.1822.

4-(1-Butyl-1H-1,2,3-triazol-4-yl)butylamine (6). In a $100 \mathrm{~mL}$ roundbottom flask, phthalimide 5 (1.10 g, $3.38 \mathrm{mmol})$ was dissolved in anhydrous THF $(5 \mathrm{~mL})$ at rt. Hydrazine hydrate $(1.41 \mathrm{~mL}, 28.9 \mathrm{mmol})$ was added and the mixture was stirred at $40{ }^{\circ} \mathrm{C}$ for $6 \mathrm{~h}$. Upon completion of the reaction, the reaction mixture was diluted with $\mathrm{Et}_{2} \mathrm{O}(70 \mathrm{~mL})$ and insoluble solid was filtered off. The filtrate was washed with aqueous $10 \% \mathrm{NaOH}(3 \mathrm{~mL})(\mathrm{pH} 12)$, and dried over anhydrous $\mathrm{Na} 2 \mathrm{SO} 4$, filtered, and concentrated in vacuo to yield analytically pure amine 6 as a colorless liquid (648 mg,98\%). TLC: Rf 0.02 (1:1 hexanes/EtOAc). ${ }^{1} \mathrm{H}$ NMR $\left(400 \mathrm{MHz} \mathrm{CDCl}_{3}\right): \delta 7.26(\mathrm{~s}, 1 \mathrm{H}), 4.31(\mathrm{t}, J=7.2 \mathrm{~Hz}, 2 \mathrm{H}), 2.73(\mathrm{t}, J=7.6 \mathrm{~Hz}$, $2 \mathrm{H}), 2.72(\mathrm{t}, J=7.2 \mathrm{~Hz}, 2 \mathrm{H}), 1.87$ (quintet, $J=7.2 \mathrm{~Hz}, 2 \mathrm{H}), 1.72$ (quintet, $J=7.6 \mathrm{~Hz}, 2 \mathrm{H}), 1.52(\mathrm{~m}, 2 \mathrm{H}$ ), 1.35 (sextet, $J=7.2 \mathrm{~Hz}, 2 \mathrm{H}), 0.95(\mathrm{t}, J=7.2 \mathrm{~Hz}, 3 \mathrm{H}) .13 \mathrm{C} \mathrm{NMR}\left(100 \mathrm{MHz}, \mathrm{CDCl}^{3}\right): \delta 147.9,120.4,49.8$, 41.9, 33.3, 32.3, 26.7, 25.5, 19.7, 13.4. LRMS (ESI) m/z (rel int): (pos) 219 ([M + Na] $\left.]^{+}, 100\right), 180$ ([M $\left.\left.\mathrm{NH}_{2}\right]^{+}, 100\right)$. HRMS (ESI) m/z calcd for C10H20N4Na ${ }^{+}\left([\mathrm{M}+\mathrm{Na}]^{+}\right)$219.1580, found 219.1578.

(S)-tert-Butyl [1-((4-(1-butyl-1H-1,2,3-triazol-4-yl)butyl)amino)-1-oxo-3-phenylpropan-2-yl]carbamate (7a). In a $50 \mathrm{~mL}$ roundbottom flask, amine $6(261 \mathrm{mg}, 1.33 \mathrm{mmol})$, Boc-L-Phe-OH (353 mg, $1.33 \mathrm{mmol})$, EDCI.HCl (286 mg, $1.46 \mathrm{mmol})$, and HOBt.hydrate $(198 \mathrm{mg}, 1.46 \mathrm{mmol})$ were dissolved in anhydrous $\mathrm{CH}_{2} \mathrm{Cl}_{2}(5.8 \mathrm{~mL})$ at $\mathrm{rt}$. The mixture was stirred at $\mathrm{rt}$ for $5 \mathrm{~h}$. Upon completion of the reaction, $10 \% \mathrm{wt}$ citric acid $(11 \mathrm{~mL})$ was added and the two layers were separated. The aqueous layer was extracted with $\mathrm{CH}_{2} \mathrm{Cl}_{2}(2 \times 30 \mathrm{~mL})$ and combined organic extracts were washed with saturated aqueous $\mathrm{NaHCO}_{3}$ $(15 \mathrm{~mL})$ and brine $(15 \mathrm{~mL})$ sequentially. It was then dried over anhydrous $\mathrm{MgSO}_{4}$, filtered, and concentrated in vacuo. The purification by column chromatography on silica gel $\left(20: 1 \mathrm{CH}_{2} \mathrm{Cl}_{2} / \mathrm{MeOH}\right)$ afforded a desired amide $7 \mathrm{a}$ as a white foam $(566 \mathrm{mg}, 96 \%)$. TLC: $\mathrm{R}_{f} 0.19$ (1:2 hexanes/EtOAc). mp: 98.8-100.8 ${ }^{\circ} \mathrm{C} .[\alpha]_{\mathrm{D}}^{21.4}=+4.2\left(\mathrm{c} 1.0, \mathrm{CHCl}_{3}\right) .{ }^{1} \mathrm{H}$ NMR $\left(400 \mathrm{MHz}, \mathrm{CDCl}_{3}\right): \delta 7.30-7.19(\mathrm{~m}, 6 \mathrm{H}), 5.82(\mathrm{br} \mathrm{s}$, $1 \mathrm{H}), 5.05$ (br s, 1H), $4.31(\mathrm{t}, J=7.2 \mathrm{~Hz}, 2 \mathrm{H}), 4.27(\mathrm{q}, J=7.6 \mathrm{~Hz}, 1 \mathrm{H}), 3.19(\mathrm{q}, J=6.4 \mathrm{~Hz}, 2 \mathrm{H}), 3.07$ (dd, $J=13.6,6.4 \mathrm{~Hz}, 1 \mathrm{H}), 3.02(\mathrm{dd}, J=13.6,7.6 \mathrm{~Hz}, 1 \mathrm{H}), 2.67(\mathrm{t}, J=7.6 \mathrm{~Hz}, 2 \mathrm{H}), 1.88$ (quintet, $J=7.6 \mathrm{~Hz}, 2 \mathrm{H}$ ), 
1.58 (quintet, $J=7.2 \mathrm{~Hz}, 2 \mathrm{H}), 1.45(\mathrm{~m}, 2 \mathrm{H}), 1.40(\mathrm{~s}, 9 \mathrm{H}), 1.36$ (sextet, $J=7.2 \mathrm{~Hz}, 2 \mathrm{H}), 0.96(\mathrm{t}, J=7.2 \mathrm{~Hz}$, $3 \mathrm{H}) .{ }^{13} \mathrm{C}$ NMR $\left(100 \mathrm{MHz}, \mathrm{CDCl}_{3}\right): \delta 171.3,155.6,147.7,137.0,129.5,128.8,127.1,120.7,80.3,56.2,50.1$, 39.3, 39.0, 32.5, 28.9, 28.4, 26.7, 25.2, 19.9, 13.7. LRMS (ESI) $\mathrm{m} / \mathrm{z}$ (rel int): (pos) 444 ([M + H] $]^{+}, 100$ ), 388 $\left(\left[\mathrm{M}-\mathrm{C}_{4} \mathrm{H}_{7}\right]^{+}, 75\right)$. HRMS (ESI) $\mathrm{m} / \mathrm{z}$ calcd for C24H38N5O3+ $\left([\mathrm{M}+\mathrm{H}]^{+}\right) 444.2969$, found 444.2977.

(R)-tert-Butyl [1-((4-(1-butyl-1H-1,2,3-triazol-4-yl)butyl)amino)-1-oxo-3-phenylpropan-2-yl]carbamate (7a'). $7 \mathbf{a}^{\prime}$ was synthesized according to the procedure described for the synthesis of $7 \mathbf{a}$. Amine $6(719 \mathrm{mg}$, $3.66 \mathrm{mmol})$, Boc-D-Phe-OH (991 mg, $3.66 \mathrm{mmol})$, EDCI.HCl (772 mg, $4.03 \mathrm{mmol})$, and HOBt.hydrate (544 mg, $4.03 \mathrm{mmol}$ ) were used. Reaction time: $6 \mathrm{~h}$. Yield: $1.60 \mathrm{~g}, 98 \%$. White solid. TLC: $\mathrm{R}_{f} 0.19$ (1:2 hexanes/EtOAc). mp: 107.1-109.1 ${ }^{\circ} \mathrm{C} .[\alpha]_{\mathrm{D}}^{22.5}=-5.7$ (c 1.0, $\left.\mathrm{CHCl}_{3}\right) .{ }^{1} \mathrm{H} \mathrm{NMR}\left(400 \mathrm{MHz}, \mathrm{CDCl}_{3}\right): \delta$ 7.30-7.19 (m, 6H), $5.89(\mathrm{br} \mathrm{s}, 1 \mathrm{H}), 5.08(\mathrm{br} \mathrm{s}, 1 \mathrm{H}), 4.31(\mathrm{t}, J=7.2 \mathrm{~Hz}, 2 \mathrm{H}), 4.28(\mathrm{q}, J=7.6 \mathrm{~Hz}, 1 \mathrm{H}), 3.19(\mathrm{q}$, $J=6.8 \mathrm{~Hz}, 2 \mathrm{H}), 3.07(\mathrm{dd}, J=13.6,6.4 \mathrm{~Hz}, 1 \mathrm{H}), 3.02(\mathrm{dd}, J=13.6,7.6 \mathrm{~Hz}, 1 \mathrm{H}), 2.67(\mathrm{t}, J=7.2 \mathrm{~Hz}, 2 \mathrm{H}), 1.87$ (quintet, $J=7.2 \mathrm{~Hz}, 2 \mathrm{H}$ ), 1.59 (quintet, $J=7.6 \mathrm{~Hz}, 2 \mathrm{H}), 1.44(\mathrm{~m}, 2 \mathrm{H}), 1.40(\mathrm{~s}, 9 \mathrm{H}), 1.36$ (sextet, $J=7.2 \mathrm{~Hz}$, $2 \mathrm{H}), 0.96(\mathrm{t}, J=7.2 \mathrm{~Hz}, 3 \mathrm{H}) .{ }^{13} \mathrm{C} \mathrm{NMR}\left(100 \mathrm{MHz}, \mathrm{CDCl}_{3}\right): \delta 171.0,155.4,147.5,136.9,129.3,128.6,126.9$, $120.5,80.1,56.1,49.9,39.1,38.8,32.3,28.8,28.3,26.5,25.1,19.7,13.5$. LRMS (ESI) $\mathrm{m} / z$ (rel int): (pos) 444 $\left([\mathrm{M}+\mathrm{H}]^{+}, 100\right), 388\left(\left[\mathrm{M}-\mathrm{C}_{4} \mathrm{H}_{7}\right]^{+}, 23\right)$. HRMS (ESI) $\mathrm{m} / \mathrm{z}$ calcd for C24H38N5O3 ${ }^{+}\left([\mathrm{M}+\mathrm{H}]^{+}\right) 444.2969$, found 444.2969 .

(S)-tert-Butyl [1-((4-(1-butyl-1H-1,2,3-triazol-4-yl)butyl)amino)-3,3-dimethyl-1-oxobutan-2-yl]carbamate (7b). $7 \mathbf{b}$ was synthesized according to the procedure described for the synthesis of $7 \mathbf{a}$. Amine $6(813 \mathrm{mg}$, $4.14 \mathrm{mmol})$, Boc-tert-Leu-OH ( $931 \mathrm{mg}, 3.95 \mathrm{mmol}), \mathrm{EDCI} \cdot \mathrm{HCl}(832 \mathrm{mg}, 4.34 \mathrm{mmol})$, and HOBt-hydrate ( $587 \mathrm{mg}$, $4.34 \mathrm{mmol}$ ) were used. Reaction time: $14 \mathrm{~h}$. Yield: $1.52 \mathrm{~g}$, $94 \%$. Colorless oil. TLC: $\mathrm{R}_{f} 0.43$ $\left(10: 1 \mathrm{CH}_{2} \mathrm{Cl}_{2} / \mathrm{MeOH}\right) .[\alpha]_{\mathrm{D}}^{22.7}=+1.3\left(\mathrm{c} 1.0, \mathrm{CHCl}_{3}\right) .{ }^{1} \mathrm{H} \mathrm{NMR}\left(400 \mathrm{MHz}, \mathrm{CDCl}_{3}\right): \delta 7.27(\mathrm{~s}, 1 \mathrm{H}), 5.78(\mathrm{br}$ $\mathrm{s}, 1 \mathrm{H}), 5.25(\mathrm{br} \mathrm{d}, J=8.8 \mathrm{~Hz}, 1 \mathrm{H}), 4.31(\mathrm{t}, J=7.2 \mathrm{~Hz}, 2 \mathrm{H}), 3.75(\mathrm{br} \mathrm{d}, J=9.2 \mathrm{~Hz}, 1 \mathrm{H}), 3.29(\mathrm{~m}, 2 \mathrm{H}), 2.73(\mathrm{t}$, $J=7.2 \mathrm{~Hz}, 2 \mathrm{H}$ ), 1.87 (quintet, $J=7.2 \mathrm{~Hz}, 2 \mathrm{H}), 1.72$ (quintet, $J=7.2 \mathrm{~Hz}, 2 \mathrm{H}), 1.59(\mathrm{~m}, 2 \mathrm{H}), 1.43(\mathrm{~s}, 9 \mathrm{H})$, 1.35 (sextet, $J=7.2 \mathrm{~Hz}, 2 \mathrm{H}), 0.98(\mathrm{~s}, 9 \mathrm{H}), 0.96(\mathrm{t}, J=7.2 \mathrm{~Hz}, 3 \mathrm{H}) .{ }^{13} \mathrm{C}$ NMR $\left(100 \mathrm{MHz}, \mathrm{CDCl}_{3}\right): \delta 170.9$, $155.8,147.5,120.6,79.6,62.5,49.9,39.1,34.5,32.3,28.9,28.3,26.7,26.6,25.1,19.7,13.5$. LRMS (ESI) $\mathrm{m} / z$ (rel int): (pos) $410\left([\mathrm{M}+\mathrm{H}]^{+}, 100\right), 354\left(\left[\mathrm{M}-\mathrm{C}_{4} \mathrm{H}_{7}\right]^{+}, 22\right)$. HRMS (ESI) $\mathrm{m} / \mathrm{z}$ calcd for $\mathrm{C} 21 \mathrm{H} 40 \mathrm{~N} 5 \mathrm{O}^{+}$ $\left([\mathrm{M}+\mathrm{H}]^{+}\right)$410.3126, found 410.3129 .

(S)-tert-Butyl [1-((4-(1-butyl-1H-1,2,3-triazol-4-yl)butyl)amino)-3-methyl-1-oxobutan-2-yl]carbamate (7c). $7 \mathrm{c}$ was synthesized according to the procedure described for the synthesis of 7a. Amine 6 (690 mg, $3.51 \mathrm{mmol})$, Boc-L-Val-OH (727 mg, $3.35 \mathrm{mmol}), \mathrm{EDCI} \cdot \mathrm{HCl}(706 \mathrm{mg}, 3.68 \mathrm{mmol})$, and HOBt.hydrate ( $498 \mathrm{mg}, 3.68 \mathrm{mmol}$ ) were used. The crude mixture was purified by column chromatography on silica gel (30:1 $\left.\mathrm{CH}_{2} \mathrm{Cl}_{2} / \mathrm{MeOH}\right)$. Reaction time: $8 \mathrm{~h}$. Yield: $1.26 \mathrm{~g}$, 95\%. White solid. TLC: $\mathrm{R}_{f} 0.52$ (10:1 $\left.\mathrm{CH}_{2} \mathrm{Cl}_{2} / \mathrm{MeOH}\right) . \mathrm{mp}: 81.5-83.5^{\circ} \mathrm{C}$. $[\alpha]_{\mathrm{D}}^{21.6}=-15.4$ (c 1.0, MeOH). ${ }^{1} \mathrm{H}$ NMR $\left(400 \mathrm{MHz}, \mathrm{CDCl}_{3}\right): \delta 7.27(\mathrm{~s}$, $1 \mathrm{H}), 6.03(\mathrm{br} \mathrm{s}, 1 \mathrm{H}), 5.02(\mathrm{br} \mathrm{s}, 1 \mathrm{H}), 4.31(\mathrm{t}, J=7.2 \mathrm{~Hz}, 2 \mathrm{H}), 3.84(\mathrm{dd}, J=8.8,6.0 \mathrm{~Hz}, 1 \mathrm{H}), 3.29(\mathrm{q}, J=6.8 \mathrm{~Hz}$, $2 \mathrm{H}), 2.73(\mathrm{t}, J=7.6 \mathrm{~Hz}, 2 \mathrm{H}), 2.14(\mathrm{~m}, 1 \mathrm{H}), 1.87$ (quintet, $J=7.2 \mathrm{~Hz}, 2 \mathrm{H}), 1.72$ (quintet, $J=7.6 \mathrm{~Hz}, 2 \mathrm{H}$ ), $1.59(\mathrm{~m}, 2 \mathrm{H}), 1.44(\mathrm{~s}, 9 \mathrm{H}), 1.35$ (sextet, $J=7.2 \mathrm{~Hz}, 2 \mathrm{H}), 0.96(\mathrm{t}, J=7.2 \mathrm{~Hz}, 3 \mathrm{H}), 0.95(\mathrm{~d}, J=6.8 \mathrm{~Hz}, 3 \mathrm{H})$, $0.90(\mathrm{~d}, J=6.8 \mathrm{~Hz}, 3 \mathrm{H}) .{ }^{13} \mathrm{C} \mathrm{NMR}\left(100 \mathrm{MHz}, \mathrm{CDCl}_{3}\right): \delta 171.6,155.9,147.6,120.6,79.8,60.1,49.9,39.1$, $32.3,30.9,28.9,28.3,26.7,25.1,19.7,19.3,17.8,13.5$. LRMS (ESI) $m / z$ (rel int): (pos) 396 ([M + H] ${ }^{+}, 100$ ), $340\left(\left[\mathrm{M}-\mathrm{C}_{4} \mathrm{H}_{7}\right]^{+}, 45\right)$. HRMS (ESI) $\mathrm{m} / \mathrm{z}$ calcd for C20H38N5O3 ${ }^{+}\left([\mathrm{M}+\mathrm{H}]^{+}\right)$396.2969, found 396.2961.

(S)-2-Amino-N-[4-(1-butyl-1H-1,2,3-triazol-4-yl)butyl]-3-phenylpropanamide (8a). In a $250 \mathrm{~mL}$ roundbottom flask, Boc protected amine $7 \mathrm{a}(1.87 \mathrm{~g}, 4.21 \mathrm{mmol})$ was dissolved in $\mathrm{CH}_{2} \mathrm{Cl}_{2}(33 \mathrm{~mL})$ at rt under air. A solution of TFA in $\mathrm{CH}_{2} \mathrm{Cl}_{2}(20 \% v / v, 48 \mathrm{~mL})$ was added dropwise for $2 \mathrm{~h}$. Upon completion of the reaction, the reaction mixture was diluted with $\mathrm{CH}_{2} \mathrm{Cl}_{2}(30 \mathrm{~mL})$, cooled to $0{ }^{\circ} \mathrm{C}$, and basified with saturated aqueous $\mathrm{NaHCO}_{3}(800 \mathrm{~mL})$. The aqueous layer was extracted with $\mathrm{CH}_{2} \mathrm{Cl}_{2}(3 \times 100 \mathrm{~mL})$. The combined organic extracts were then dried over anhydrous $\mathrm{Na}_{2} \mathrm{SO}_{4}$, filtered, and concentrated in vacuo to yield analytically pure amine $8 \mathrm{a}$ as a white solid (1.52 g, quantitative yield). TLC: $\mathrm{R}_{f} 0.65$ (5:1 $\left.\mathrm{CH}_{2} \mathrm{Cl}_{2} / \mathrm{MeOH}\right) . \mathrm{mp}: 57.2-59.2{ }^{\circ} \mathrm{C} .[\alpha]_{\mathrm{D}}^{21.6}=-44.3\left(\mathrm{c} 1.0, \mathrm{CHCl}_{3}\right) .{ }^{1} \mathrm{H}$ NMR $\left(400 \mathrm{MHz}, \mathrm{CDCl}_{3}\right): \delta$ 7.33-7.21 (m, 6H), $4.31(\mathrm{t}, J=7.2 \mathrm{~Hz}, 2 \mathrm{H}), 3.62(\mathrm{dd}, J=9.2,4.0 \mathrm{~Hz}, 1 \mathrm{H}), 3.31-3.25(\mathrm{~m}, 3 \mathrm{H}), 2.73(\mathrm{t}, J=7.2$ 
$\mathrm{Hz}, 2 \mathrm{H}), 2.71(\mathrm{dd}, J=13.2,9.2 \mathrm{~Hz}, 1 \mathrm{H}), 1.87$ (quintet, $J=7.2 \mathrm{~Hz}, 2 \mathrm{H}), 1.70$ (quintet, $J=7.2 \mathrm{~Hz}, 2 \mathrm{H}$ ), $1.57(\mathrm{~m}, 2 \mathrm{H}), 1.36$ (sextet, $J=7.2 \mathrm{~Hz}, 2 \mathrm{H}), 0.96(\mathrm{t}, J=7.2 \mathrm{~Hz}, 3 \mathrm{H}) .{ }^{13} \mathrm{C}$ NMR $\left(100 \mathrm{MHz}, \mathrm{CDCl}_{3}\right): \delta 174.3$, 147.8, 138.1, 129.4, 128.8, 126.9, 120.7, 56.6, 50.0, 41.2, 38.9, 32.4, 29.2, 26.9, 25.3, 19.9, 13.6. LRMS (ESI) $m / z$ (rel int): (pos) 344 ([M $\left.+\mathrm{H}]^{+}, 100\right), 180$ ([M $\left.\left.-n-\mathrm{Bu}-\mathrm{NH}_{2}-\mathrm{Bn}\right]^{+}, 33\right)$. HRMS (ESI) $\mathrm{m} / \mathrm{z}$ calcd for $\mathrm{C} 19 \mathrm{H} 30 \mathrm{~N}^{2} \mathrm{O}^{+}\left([\mathrm{M}+\mathrm{H}]^{+}\right) 344.2445$, found 344.2442 .

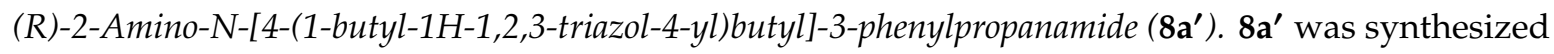
according to the procedure described for the synthesis of $8 \mathbf{a}$. Amine $7 \mathbf{a}^{\prime}(818 \mathrm{mg}, 1.84 \mathrm{mmol})$ and a solution of TFA in anhydrous $\mathrm{CH}_{2} \mathrm{Cl}_{2}(20 \% v / v, 16 \mathrm{~mL})$ were used. Reaction time: $1 \mathrm{~h}$. Yield: $615 \mathrm{mg}$, 97\%. White solid. TLC: $\mathrm{R}_{f} 0.65\left(5: 1 \mathrm{CH}_{2} \mathrm{Cl}_{2} / \mathrm{MeOH}\right)$. mp: $62.5-64.5^{\circ} \mathrm{C}$. $[\alpha]_{\mathrm{D}}^{20.6}=-16.9$ (c 1.0, $\left.\mathrm{MeOH}\right)$. ${ }^{1} \mathrm{H} \mathrm{NMR}\left(400 \mathrm{MHz}, \mathrm{CDCl}_{3}\right): \delta 7.33-7.21(\mathrm{~m}, 6 \mathrm{H}), 4.32(\mathrm{t}, J=7.2 \mathrm{~Hz}, 2 \mathrm{H}), 3.59(\mathrm{dd}, J=9.2,4.0 \mathrm{~Hz}, 1 \mathrm{H})$, $3.29(\mathrm{t}, J=7.2 \mathrm{~Hz}, 2 \mathrm{H}), 3.27(\mathrm{dd}, J=13.6,4.0 \mathrm{~Hz}, 1 \mathrm{H}), 2.73(\mathrm{t}, J=7.2 \mathrm{~Hz}, 2 \mathrm{H}), 2.68(\mathrm{dd}, J=13.6,9.2 \mathrm{~Hz}$, $1 \mathrm{H}$ ), 1.87 (quintet, $J=7.2 \mathrm{~Hz}, 2 \mathrm{H}$ ), 1.70 (quintet, $J=7.2 \mathrm{~Hz}, 2 \mathrm{H}$ ), $1.56(\mathrm{~m}, 2 \mathrm{H}), 1.36$ (sextet, $J=7.2 \mathrm{~Hz}$, 2H), $0.96(\mathrm{t}, J=7.2 \mathrm{~Hz}, 3 \mathrm{H}) .{ }^{13} \mathrm{C} \mathrm{NMR}\left(100 \mathrm{MHz}, \mathrm{CDCl}_{3}\right): \delta 174.2,147.7,138.0,129.3,128.7,126.8,120.6$, 56.6, 49.9, 41.1, 38.8, 32.3, 29.1, 26.8, 25.2, 19.7, 13.5. LRMS (ESI) $\mathrm{m} / z$ (rel int): (pos) 344 ([M + H $]^{+}, 100$ ), $180\left(\left[\mathrm{M}-\mathrm{C}_{4} \mathrm{H}_{9}-\mathrm{NH}_{2}-\mathrm{Bn}\right]^{+}\right.$, 37). HRMS (ESI) m/z calcd for $\mathrm{C} 19 H 30 \mathrm{~N}^{+} \mathrm{O}^{+}\left([\mathrm{M}+\mathrm{H}]^{+}\right) 344.2445$, found 344.2445 .

(S)-2-Amino-N-[4-(1-butyl-1H-1,2,3-triazol-4-yl)butyl]-3,3-dimethylbutanamide (8b). 8b was synthesized according to the procedure described for the synthesis of $8 \mathrm{a}$. Amine $7 \mathrm{~b}(1.16 \mathrm{~g}, 2.84 \mathrm{mmol})$ and a solution of TFA in anhydrous $\mathrm{CH}_{2} \mathrm{Cl}_{2}(20 \% \mathrm{v} / \mathrm{v}, 30 \mathrm{~mL})$ were used. Reaction time: $1.5 \mathrm{~h}$. Yield: $871 \mathrm{mg}$, 99\%. White solid. TLC: $\mathrm{R}_{f} 0.17\left(10: 1 \mathrm{CH}_{2} \mathrm{Cl}_{2} / \mathrm{MeOH}\right) . \mathrm{mp}: 45.0-47.0^{\circ} \mathrm{C}$. $[\alpha]_{\mathrm{D}}^{21.8}=+27.8($ c $1.0, \mathrm{MeOH})$. ${ }^{1} \mathrm{H} \mathrm{NMR}\left(400 \mathrm{MHz} \mathrm{CDCl}_{3}\right): \delta 7.27(\mathrm{~s}, 1 \mathrm{H}), 6.81(\mathrm{br} \mathrm{s}, 1 \mathrm{H}), 4.31(\mathrm{t}, J=7.2 \mathrm{~Hz}, 2 \mathrm{H}), 3.29(\mathrm{~m}, 2 \mathrm{H}), 3.09(\mathrm{~s}$, $1 \mathrm{H}), 2.74(\mathrm{t}, J=7.2 \mathrm{~Hz}, 2 \mathrm{H}), 1.87$ (quintet, $J=7.2 \mathrm{~Hz}, 2 \mathrm{H}), 1.73$ (quintet, $J=7.2 \mathrm{~Hz}, 2 \mathrm{H}), 1.58(\mathrm{~m}, 2 \mathrm{H}$ ), 1.35 (sextet, $J=7.2 \mathrm{~Hz}, 2 \mathrm{H}), 0.99(\mathrm{~s}, 9 \mathrm{H}), 0.95(\mathrm{t}, J=7.2 \mathrm{~Hz}, 3 \mathrm{H}) .{ }^{13} \mathrm{C} \mathrm{NMR}\left(100 \mathrm{MHz}, \mathrm{CDCl}_{3}\right): \delta 173.5$, 147.6, 120.6, 64.4, 49.9, 38.7, 34.0, 32.3, 29.1, 26.8, 26.7, 25.2, 19.7, 13.5. LRMS (ESI) $\mathrm{m} / \mathrm{z}$ (rel int): (pos) 310 $\left([\mathrm{M}+\mathrm{H}]^{+}, 100\right), 180\left(\left[\mathrm{M}-n-\mathrm{Bu}-\mathrm{NH}_{2}-t-\mathrm{Bu}\right]^{+}, 37\right)$. HRMS (ESI) $\mathrm{m} / \mathrm{z}$ calcd for $\mathrm{C} 16 \mathrm{H} 32 \mathrm{~N} 5 \mathrm{O}^{+}([\mathrm{M}+$ $\mathrm{H}]^{+}$) 310.2601, found 310.2605.

(S)-2-Amino-N-[4-(1-butyl-1H-1,2,3-triazol-4-yl)butyl]-3-methylbutanamide (8c). 8c was synthesized according to the procedure described for the synthesis of 8a. Amine 7c $(933 \mathrm{mg}, 2.36 \mathrm{mmol})$ and a solution of TFA in anhydrous $\mathrm{CH}_{2} \mathrm{Cl}_{2}(20 \% v / v, 20 \mathrm{~mL})$ were used. Reaction time: $2.5 \mathrm{~h}$. Yield: $687 \mathrm{mg}$, 99\%. Ivory solid. TLC: $\mathrm{R}_{f} 0.33\left(10: 1 \mathrm{CH}_{2} \mathrm{Cl}_{2} / \mathrm{MeOH}\right) . \mathrm{mp}: 58.9-60.9^{\circ} \mathrm{C}$. $[\alpha]_{\mathrm{D}}^{21.9}=+5.9$ (c $\left.1.0, \mathrm{MeOH}\right)$. ${ }^{1} \mathrm{H}$ NMR $\left(400 \mathrm{MHz}, \mathrm{CDCl}_{3}\right): \delta 7.36(\mathrm{br} \mathrm{s}, 1 \mathrm{H}), 7.27(\mathrm{~s}, 1 \mathrm{H}), 4.31(\mathrm{t}, J=7.2 \mathrm{~Hz}, 2 \mathrm{H}), 3.31(\mathrm{~m}, 2 \mathrm{H}), 3.23$ $(\mathrm{d}, J=3.6 \mathrm{~Hz}, 1 \mathrm{H}), 2.74(\mathrm{t}, J=7.6 \mathrm{~Hz}, 2 \mathrm{H}), 2.30(\mathrm{~m}, 1 \mathrm{H}), 1.87$ (quintet, $J=7.2 \mathrm{~Hz}, 2 \mathrm{H}), 1.73$ (quintet, $J=7.6 \mathrm{~Hz}, 2 \mathrm{H}), 1.58(\mathrm{~m}, 2 \mathrm{H}), 1.35$ (sextet, $J=7.2 \mathrm{~Hz}, 2 \mathrm{H}), 0.98(\mathrm{~d}, J=6.8 \mathrm{~Hz}, 3 \mathrm{H}), 0.95(\mathrm{t}, J=7.2 \mathrm{~Hz}, 3 \mathrm{H})$, $0.81(\mathrm{~d}, J=6.8 \mathrm{~Hz}, 3 \mathrm{H}) .{ }^{13} \mathrm{C} \mathrm{NMR}\left(100 \mathrm{MHz}, \mathrm{CDCl}_{3}\right): \delta 174.2,147.7,120.6,60.2,49.9,38.7,32.3,30.8$, 29.2, 26.8, 25.2, 19.8, 19.7, 16.1, 13.5. LRMS (ESI) $m / z$ (rel int): (pos) $296\left([\mathrm{M}+\mathrm{H}]^{+}, 100\right), 180$ ([M - n-Bu $\left.-\mathrm{NH}_{2}-i-\mathrm{Pr}\right]^{+}$, 53). HRMS (ESI) $\mathrm{m} / \mathrm{z}$ calcd for C15H30N5O ${ }^{+}\left([\mathrm{M}+\mathrm{H}]^{+}\right)$296.2445, found 296.2450.

(S)-N1-[4-(1-Butyl-1H-1,2,3-triazol-4-yl)butyl]-3-phenylpropane-1,2-diamine (9a). In a $100 \mathrm{~mL}$ roundbottom flask, amide $8 \mathrm{a}(1.40 \mathrm{~g}, 4.09 \mathrm{mmol})$ was dissolved in anhydrous THF $(16 \mathrm{~mL})$ at $\mathrm{rt}$. The reaction mixture was cooled to $0{ }^{\circ} \mathrm{C}$ and $\mathrm{LiAlH}_{4}(931 \mathrm{mg}, 24.5 \mathrm{mmol})$ was added. It was then stirred at $0{ }^{\circ} \mathrm{C}$ for $30 \mathrm{~min}$ and heated under reflux for $7 \mathrm{~h}$. Upon completion of the reaction, the resulting grey suspension was cooled to $0{ }^{\circ} \mathrm{C}$, quenched with saturated aqueous $\mathrm{Na}_{2} \mathrm{SO}_{4}$, and diluted with EtOAc $(70 \mathrm{~mL})$. The precipitate was filtered off, and the filtrate was concentrated by rotary evaporation. The residue was diluted with $\mathrm{CH}_{2} \mathrm{Cl}_{2}(100 \mathrm{~mL})$, dried over anhydrous $\mathrm{K}_{2} \mathrm{CO}_{3}$, filtered, and concentrated in vacuo. The purification by column chromatography on silica gel $\left(15: 1 \mathrm{CH}_{2} \mathrm{Cl}_{2} / \mathrm{MeOH} \rightarrow 10: 1 \mathrm{CH}_{2} \mathrm{Cl}_{2} / \mathrm{MeOH} \rightarrow \mathrm{MeOH}\right)$ afforded a desired diamine $9 \mathrm{a}$ as a pale yellow solid $(1.17 \mathrm{~g}, 87 \%)$. TLC: $\mathrm{R}_{f} 0.04(\mathrm{MeOH}) . \mathrm{mp}: 38.1-40.1{ }^{\circ} \mathrm{C}$. $[\alpha]_{\mathrm{D}}^{21.0}=+6.3(\mathrm{c} 1.0, \mathrm{MeOH}) .{ }^{1} \mathrm{H}$ NMR $\left(400 \mathrm{MHz} \mathrm{CDCl}_{3}\right): \delta 7.32-7.18(\mathrm{~m}, 6 \mathrm{H}), 4.31(\mathrm{t}, J=7.2 \mathrm{~Hz}, 2 \mathrm{H})$, $3.09(\mathrm{ddd}, J=13.6,8.8,4.8 \mathrm{~Hz}, 1 \mathrm{H}), 2.78(\mathrm{dd}, J=13.6,4.8 \mathrm{~Hz}, 1 \mathrm{H}), 2.73(\mathrm{t}, J=7.2 \mathrm{~Hz}, 2 \mathrm{H}), 2.70(\mathrm{dd}$, $J=11.6,4.8 \mathrm{~Hz}, 1 \mathrm{H}), 2.64(\mathrm{q}, J=7.2 \mathrm{~Hz}, 2 \mathrm{H}), 2.49(\mathrm{dd}, J=13.6,8.8 \mathrm{~Hz}, 1 \mathrm{H}), 2.45(\mathrm{dd}, J=11.6,8.8 \mathrm{~Hz}$, 
$1 \mathrm{H}$ ), 1.87 (quintet, $J=7.2 \mathrm{~Hz}, 2 \mathrm{H}$ ), 1.72 (quintet, $J=7.2 \mathrm{~Hz}, 2 \mathrm{H}$ ), $1.55(\mathrm{~m}, 2 \mathrm{H}), 1.35$ (sextet, $J=7.2 \mathrm{~Hz}$, $2 \mathrm{H}), 0.95(\mathrm{t}, J=7.2 \mathrm{~Hz}, 3 \mathrm{H}) .{ }^{13} \mathrm{C} \mathrm{NMR}\left(100 \mathrm{MHz}, \mathrm{CD}_{3} \mathrm{OD}\right): \delta 149.0,140.2,130.4,129.6,127.5,123.1,56.3$, 53.3, 51.0, 50.5, 43.2, 33.4, 30.0, 28.3, 26.1, 20.7, 13.8. LRMS (ESI) $\mathrm{m} / z$ (rel int): (pos) $330\left([\mathrm{M}+\mathrm{H}]^{+}\right.$, 100), $282\left(\left[\mathrm{C}_{15} \mathrm{H}_{32} \mathrm{~N}_{5}\right]^{+}, 11\right), 180\left(\left[\mathrm{M}-\mathrm{C}_{2} \mathrm{H}_{6} \mathrm{~N}_{2}-\mathrm{Bn}\right]^{+}, 23\right)$. HRMS (ESI) $\mathrm{m} / \mathrm{z}$ calcd for $\mathrm{C} 19 \mathrm{H} 32 \mathrm{~N} 5+$ $\left([\mathrm{M}+\mathrm{H}]^{+}\right) 330.2652$, found 330.2659 .

(R)-N1-[4-(1-Butyl-1H-1,2,3-triazol-4-yl)butyl]-3-phenylpropane-1,2-diamine (9a'). 9a' was synthesized according to the procedure described for the synthesis of 9a. Amide 8a' (425 mg, $1.24 \mathrm{mmol}$ ) and $\mathrm{LiAlH}_{4}(299 \mathrm{mg}, 7.88 \mathrm{mmol})$ were used. Reaction time: $6 \mathrm{~h}$. Yield: $361 \mathrm{mg}, 88 \%$. White solid. TLC: $\mathrm{R}_{f}$ $0.04(\mathrm{MeOH}) . \mathrm{mp}: 47.2-49.2^{\circ} \mathrm{C}$. $[\alpha]_{\mathrm{D}}^{21.1}=-7.6(\mathrm{c} 1.0, \mathrm{MeOH}) .{ }^{1} \mathrm{H}$ NMR $\left(400 \mathrm{MHz}, \mathrm{CDCl}_{3}\right): \delta 7.32-7.18$ $(\mathrm{m}, 6 \mathrm{H}), 4.31(\mathrm{t}, J=7.2 \mathrm{~Hz}, 2 \mathrm{H}), 3.09(\mathrm{br} \mathrm{s}, 1 \mathrm{H}), 2.78(\mathrm{dd}, J=13.2,4.8 \mathrm{~Hz}, 1 \mathrm{H}), 2.73(\mathrm{t}, J=7.6 \mathrm{~Hz}, 2 \mathrm{H})$, $2.68(\mathrm{~m}, 1 \mathrm{H}), 2.64(\mathrm{q}, J=7.2 \mathrm{~Hz}, 2 \mathrm{H}), 2.50(\mathrm{dd}, J=13.2,8.8 \mathrm{~Hz}, 1 \mathrm{H}), 2.45(\mathrm{dd}, J=11.2,8.8 \mathrm{~Hz}, 1 \mathrm{H}), 1.87$ (quintet, $J=7.2 \mathrm{~Hz}, 2 \mathrm{H}$ ), 1.72 (quintet, $J=7.6 \mathrm{~Hz}, 2 \mathrm{H}$ ), $1.55(\mathrm{~m}, 2 \mathrm{H}), 1.35$ (sextet, $J=7.2 \mathrm{~Hz}, 2 \mathrm{H}$ ), 0.95 $(\mathrm{t}, J=7.2 \mathrm{~Hz}, 3 \mathrm{H}) .{ }^{13} \mathrm{C}$ NMR $\left(100 \mathrm{MHz}, \mathrm{CD}_{3} \mathrm{OD}\right): \delta 149.0,140.2,130.4,129.6,127.5,123.2,56.4,53.3$, 51.0, 50.5, 43.2, 33.4, 30.0, 28.3, 26.1, 20.7, 13.8. LRMS (ESI) $\mathrm{m} / z$ (rel int): (pos) 330 ([M + H] $\left.{ }^{+}, 100\right), 282$ $\left(\left[\mathrm{C}_{15} \mathrm{H}_{32} \mathrm{~N}_{5}\right]^{+}, 48\right), 180\left(\left[\mathrm{M}-\mathrm{C}_{2} \mathrm{H}_{6} \mathrm{~N}_{2}-\mathrm{Bn}\right]^{+}, 22\right)$. HRMS (ESI) $\mathrm{m} / \mathrm{z}$ calcd for C19H32N5 ${ }^{+}\left([\mathrm{M}+\mathrm{H}]^{+}\right)$ 330.2652 , found 330.2658 .

(S)-N1-[4-(1-Butyl-1H-1,2,3-triazol-4-yl)butyl]-3,3-dimethylbutane-1,2-diamine (9b). 9b was synthesized according to the procedure described for the synthesis of $9 \mathbf{a}$. Amide $8 \mathbf{b}(2.30 \mathrm{~g}, 7.42 \mathrm{mmol})$ and $\mathrm{LiAlH}_{4}$ $(1.69 \mathrm{~g}, 44.5 \mathrm{mmol})$ were used. Reaction time: $24 \mathrm{~h}$. Yield: $1.47 \mathrm{~g}, 67 \%$ (brsm: 83\%). Colorless oil. TLC: $\mathrm{R}_{f} 0.07(\mathrm{MeOH}) \cdot[\alpha]_{\mathrm{D}}^{21.7}=+23.8(\mathrm{c} 1.0, \mathrm{MeOH}) .{ }^{1} \mathrm{H} \mathrm{NMR}\left(400 \mathrm{MHz}, \mathrm{CDCl}_{3}\right): \delta 7.25(\mathrm{~s}, 1 \mathrm{H}), 4.31(\mathrm{t}$, $J=7.2 \mathrm{~Hz}, 2 \mathrm{H}), 2.77(\mathrm{dd}, J=11.2,2.4 \mathrm{~Hz}, 1 \mathrm{H}), 2.74(\mathrm{t}, J=7.6 \mathrm{~Hz}, 2 \mathrm{H}), 2.67(\mathrm{dt}, J=11.2,7.2 \mathrm{~Hz}, 1 \mathrm{H}), 2.59$ $(\mathrm{dt}, J=11.2,7.2 \mathrm{~Hz}, 1 \mathrm{H}), 2.46(\mathrm{dd}, J=11.2,2.4 \mathrm{~Hz}, 1 \mathrm{H}), 2.21(\mathrm{t}, J=11.2 \mathrm{~Hz}, 1 \mathrm{H}), 1.87$ (quintet, $J=7.2 \mathrm{~Hz}$, 2H), 1.73 (quintet, $J=7.6 \mathrm{~Hz}, 2 \mathrm{H}), 1.57(\mathrm{~m}, 2 \mathrm{H}), 1.35$ (sextet, $J=7.2 \mathrm{~Hz}, 2 \mathrm{H}), 0.95(\mathrm{t}, J=7.2 \mathrm{~Hz}, 3 \mathrm{H}), 0.88$ (s, 9H). ${ }^{13} \mathrm{C}$ NMR (100 MHz, DMSO- $\left.d_{6}\right)$ : $\delta 146.8,121.5,59.1,51.3,49.2,48.8,33.3,31.7,29.3,26.9,26.3$, 25.0, 19.1, 13.3. LRMS (ESI) $m / z$ (rel int): (pos) $296\left([\mathrm{M}+\mathrm{H}]^{+}, 100\right), 282\left(\left[\mathrm{C}_{15} \mathrm{H}_{32} \mathrm{~N}_{5}\right]^{+}, 21\right)$. HRMS (ESI) $\mathrm{m} / \mathrm{z}$ calcd for $\mathrm{C} 16 \mathrm{H} 34 \mathrm{~N}^{+}\left([\mathrm{M}+\mathrm{H}]^{+}\right)$296.2809, found 296.2814 .

(S)-N1-[4-(1-Butyl-1H-1,2,3-triazol-4-yl)butyl]-3-methylbutane-1,2-diamine (9c). 9c was synthesized according to the procedure described for the synthesis of 9a. Amide 8c (900 mg, $3.05 \mathrm{mmol}$ ) and $\mathrm{LiAlH}_{4}(482 \mathrm{mg}, 18.3 \mathrm{mmol}$ ) were used. Reaction time: $24 \mathrm{~h}$. Yield: $694 \mathrm{mg}, 81 \%$ (brsm: 89\%). Milky oil. TLC: $\mathrm{R}_{f} 0.05(\mathrm{MeOH}) \cdot[\alpha]_{\mathrm{D}}^{21.8}=+16.3(\mathrm{c} 1.0, \mathrm{MeOH}) .{ }^{1} \mathrm{H}$ NMR $\left(400 \mathrm{MHz}, \mathrm{CDCl}_{3}\right): \delta 7.25(\mathrm{~s}, 1 \mathrm{H}), 4.31$ $(\mathrm{t}, J=7.2 \mathrm{~Hz}, 2 \mathrm{H}), 2.73(\mathrm{t}, J=7.6 \mathrm{~Hz}, 2 \mathrm{H}), 2.69-2.56(\mathrm{~m}, 4 \mathrm{H}), 2.34(\mathrm{dd}, J=11.6,9.6 \mathrm{~Hz}, 1 \mathrm{H}), 1.87$ (quintet, $J=7.2 \mathrm{~Hz}, 2 \mathrm{H}$ ), 1.72 (quintet, $J=7.6 \mathrm{~Hz}, 2 \mathrm{H}), 1.60-1.52(\mathrm{~m}, 3 \mathrm{H}), 1.35$ (sextet, $J=7.2 \mathrm{~Hz}, 2 \mathrm{H}), 0.95(\mathrm{t}$, $J=7.2 \mathrm{~Hz}, 3 \mathrm{H}), 0.91(\mathrm{~d}, J=6.8 \mathrm{~Hz}, 3 \mathrm{H}), 0.89(\mathrm{~d}, J=6.8 \mathrm{~Hz}, 3 \mathrm{H}) .{ }^{13} \mathrm{C} \mathrm{NMR}\left(100 \mathrm{MHz}, \mathrm{CDCl}_{3}\right): \delta 148.1$, 120.4 56.6, 54.2, 49.9 (2C), 32.5, 32.3, 29.8, 27.3, 25.6, 19.8, 19.4, 17.8, 13.5. LRMS (ESI) $m / z$ (rel int): (pos) $282\left([\mathrm{M}+\mathrm{H}]^{+}, 82\right), 141\left(\left[\mathrm{M}-\mathrm{nBu}-\mathrm{C}_{2} \mathrm{HN}_{3}-\mathrm{NH}_{2}\right]^{+}, 100\right)$. HRMS (ESI) $\mathrm{m} / \mathrm{z}$ calcd for $\mathrm{C} 15 \mathrm{H} 32 \mathrm{N5}^{+}$ $\left([\mathrm{M}+\mathrm{H}]^{+}\right)$282.2652, found 282.2662.

(S)-4-Benzyl-1-[4-(1-butyl-1H-1,2,3-triazol-4-yl)butyl]imidazolidin-2-one (10a). In a $100 \mathrm{~mL}$ roundbottom flask, diamine 9a (440 mg, $1.34 \mathrm{mmol})$ was dissolved in anhydrous DMF $(1.3 \mathrm{~mL})$ at rt. $\mathrm{Et}_{3} \mathrm{~N}(470 \mu \mathrm{L}$, $3.34 \mathrm{mmol})$ and CDI (335 mg, $2.00 \mathrm{mmol})$ were added. The resulting reaction mixture was stirred at $120^{\circ} \mathrm{C}$ for $13 \mathrm{~h}$. Upon completion of the reaction, the reaction mixture was cooled to $\mathrm{rt}$, and concentrated in vacuo. The purification by column chromatography on silica gel $\left(15: 1 \mathrm{CH}_{2} \mathrm{Cl}_{2} / \mathrm{MeOH}\right)$ afforded a desired imidazolidinone 10a as a colorless liquid (470 mg, 99\%). TLC: $\mathrm{R}_{f} 0.49$ (10:1 $\left.\mathrm{CH}_{2} \mathrm{Cl}_{2} / \mathrm{MeOH}\right)$. $[\alpha]_{\mathrm{D}}^{22.3}=-27.7\left(\mathrm{c} \mathrm{1.0}, \mathrm{CHCl}_{3}\right) .{ }^{1} \mathrm{H}$ NMR $\left(400 \mathrm{MHz}, \mathrm{CDCl}_{3}\right): \delta 7.34-7.30(\mathrm{~m}, 2 \mathrm{H}), 7.28-7.23(\mathrm{~m}, 2 \mathrm{H})$, 7.19-7.17 (m, 2H), $4.31(\mathrm{t}, J=7.2 \mathrm{~Hz}, 2 \mathrm{H}), 4.30(\mathrm{br} \mathrm{s}, 1 \mathrm{H}), 3.86(\mathrm{~m}, 1 \mathrm{H}), 3.50(\mathrm{t}, J=8.8 \mathrm{~Hz}, 1 \mathrm{H}), 3.20(\mathrm{~m}$, $2 \mathrm{H}), 3.13(\mathrm{dd}, J=8.8,6.0 \mathrm{~Hz}, 1 \mathrm{H}), 2.83(\mathrm{dd}, J=13.2,6.0 \mathrm{~Hz}, 1 \mathrm{H}), 2.77(\mathrm{dd}, J=13.2,8.8 \mathrm{~Hz}, 1 \mathrm{H}), 2.75(\mathrm{t}$, $J=7.6 \mathrm{~Hz}, 2 \mathrm{H}), 1.87$ (quintet, $J=7.2 \mathrm{~Hz}, 2 \mathrm{H}), 1.69(\mathrm{~m}, 2 \mathrm{H}), 1.57(\mathrm{~m}, 2 \mathrm{H}), 1.35$ (sextet, $J=7.2 \mathrm{~Hz}, 2 \mathrm{H}$ ), $0.95(\mathrm{t}, J=7.2 \mathrm{~Hz}, 3 \mathrm{H}) .{ }^{13} \mathrm{C} \mathrm{NMR}\left(100 \mathrm{MHz}, \mathrm{CDCl}_{3}\right): \delta 161.7,147.8,137.2,129.2,128.9,127.0,120.7,51.4$, 
50.3, 50.0, 43.0, 42.1, 32.4, 27.2, 26.7, 25.3, 19.8, 13.6. LRMS (ESI) $\mathrm{m} / z$ (rel int): (pos) 356 ([M + H] $]^{+}, 16$ ), $282\left(\left[\mathrm{C}_{15} \mathrm{H}_{32} \mathrm{~N}_{5}\right]^{+}, 100\right)$. HRMS (ESI) $\mathrm{m} / \mathrm{z}$ calcd for C20H30N5O ${ }^{+}\left([\mathrm{M}+\mathrm{H}]^{+}\right) 356.2445$, found 356.2440 .

(R)-4-Benzyl-1-[4-(1-butyl-1H-1,2,3-triazol-4-yl)butyl]imidazolidin-2-one (10a'). 10a' was synthesized according to the procedure described for the synthesis of 10a. Diamine $9 \mathbf{a}^{\prime}(179 \mathrm{mg}, 542 \mu \mathrm{mol}), \mathrm{Et}_{3} \mathrm{~N}$ $(190 \mu \mathrm{L}, 1.36 \mathrm{mmol})$ and CDI $(132 \mathrm{mg}, 814 \mu \mathrm{mol})$ were used. Reaction time: $13 \mathrm{~h}$. Yield: $175 \mathrm{mg}, 91 \%$. Colorless liquid. TLC: $\mathrm{R}_{f} 0.49\left(10: 1 \mathrm{CH}_{2} \mathrm{Cl}_{2} / \mathrm{MeOH}\right) .[\alpha]_{\mathrm{D}}^{22.8}=+26.4$ (c 1.0, $\left.\mathrm{CHCl}_{3}\right) .{ }^{1} \mathrm{H} \mathrm{NMR}(400 \mathrm{MHz}$, $\left.\mathrm{CDCl}_{3}\right): \delta 7.34-7.30(\mathrm{~m}, 2 \mathrm{H}), 7.27-7.22(\mathrm{~m}, 2 \mathrm{H}), 7.19-7.17(\mathrm{~m}, 2 \mathrm{H}), 4.31(\mathrm{t}, J=7.2 \mathrm{~Hz}, 2 \mathrm{H}), 4.29(\mathrm{br} \mathrm{s}, 1 \mathrm{H})$, $3.86(\mathrm{~m}, 1 \mathrm{H}), 3.49(\mathrm{t}, J=8.4 \mathrm{~Hz}, 1 \mathrm{H}), 3.23(\mathrm{dq}, J=14.0,7.2 \mathrm{~Hz}, 2 \mathrm{H}), 3.20(\mathrm{~m}, 2 \mathrm{H}), 3.13(\mathrm{dd}, J=8.8,6.0 \mathrm{~Hz}$, $1 \mathrm{H}), 2.82(\mathrm{~m}, 2 \mathrm{H}), 2.75(\mathrm{t}, J=7.6 \mathrm{~Hz}, 2 \mathrm{H}), 1.87$ (quintet, $J=7.2 \mathrm{~Hz}, 2 \mathrm{H}), 1.69(\mathrm{~m}, 2 \mathrm{H}), 1.56(\mathrm{~m}, 2 \mathrm{H}), 1.35$ (sextet, $J=7.2 \mathrm{~Hz}, 2 \mathrm{H}), 0.95(\mathrm{t}, J=7.2 \mathrm{~Hz}, 3 \mathrm{H}) .{ }^{13} \mathrm{C} \mathrm{NMR}\left(100 \mathrm{MHz}, \mathrm{CDCl}_{3}\right): \delta 161.7,147.8,137.3,129.2$, 129.0, 127.1, 120.7, 51.5, 50.4, 50.1, 43.1, 42.2, 32.5, 27.2, 26.8, 25.4, 19.9, 13.7. LRMS (ESI) $\mathrm{m} / \mathrm{z}$ (rel int): (pos) $356\left([\mathrm{M}+\mathrm{H}]^{+}, 100\right), 282\left(\left[\mathrm{C}_{15} \mathrm{H}_{32} \mathrm{~N}_{5}\right]^{+}, 95\right)$. HRMS (ESI) $\mathrm{m} / \mathrm{z}$ calcd for $\mathrm{C} 20 \mathrm{H} 30 \mathrm{~N}^{+} \mathrm{O}^{+}\left([\mathrm{M}+\mathrm{H}]^{+}\right)$ 356.2445 , found 356.2452 .

(S)-4-(tert-Butyl)-1-[4-(1-butyl-1H-1,2,3-triazol-4-yl)butyl]imidazolidin-2-one (10b). 10b was synthesized according to the procedure described for the synthesis of $10 \mathrm{a}$. Diamine $9 \mathrm{~b}(1.35 \mathrm{~g}, 4.55 \mathrm{mmol}), \mathrm{Et}_{3} \mathrm{~N}$ $(1.60 \mathrm{~mL}, 11.4 \mathrm{mmol})$ and CDI $(1.11 \mathrm{~g}, 6.83 \mathrm{mmol})$ were used. Reaction time: $15 \mathrm{~h}$. Yield: $1.46 \mathrm{~g}$, quantitative yield. Colorless crystal. TLC: $\mathrm{Rf} 0.29\left(30: 1 \mathrm{CH}_{2} \mathrm{Cl}_{2} / \mathrm{MeOH}(\times 2)\right) . \mathrm{mp}: 80.3-82.3{ }^{\circ} \mathrm{C}$. $[\alpha]_{\mathrm{D}}^{22.6}=-6.9\left(\mathrm{c} 1.0, \mathrm{CHCl}_{3}\right) .{ }^{1} \mathrm{H}$ NMR $\left(400 \mathrm{MHz}, \mathrm{CDCl}_{3}\right): \delta 7.27(\mathrm{~s}, 1 \mathrm{H}), 4.31(\mathrm{t}, J=7.2 \mathrm{~Hz}, 2 \mathrm{H}), 4.30(\mathrm{~s}$, $1 \mathrm{H}), 3.41-3.34(\mathrm{~m}, 2 \mathrm{H}), 3.25(\mathrm{dt}, J=13.6,7.2 \mathrm{~Hz}, 1 \mathrm{H}), 3.15(\mathrm{dd}, J=13.6,7.2 \mathrm{~Hz}, 1 \mathrm{H}), 3.13(\mathrm{~m}, 1 \mathrm{H}), 2.75(\mathrm{t}$, $J=7.6 \mathrm{~Hz}, 2 \mathrm{H}), 1.87$ (quintet, $J=7.2 \mathrm{~Hz}, 2 \mathrm{H}), 1.68(\mathrm{~m}, 2 \mathrm{H}), 1.58(\mathrm{~m}, 2 \mathrm{H}), 1.35$ (sextet, $J=7.2 \mathrm{~Hz}, 2 \mathrm{H}$ ), $0.95(\mathrm{t}, J=7.2 \mathrm{~Hz}, 3 \mathrm{H}), 0.87(\mathrm{~s}, 9 \mathrm{H}) .{ }^{13} \mathrm{C} \mathrm{NMR}\left(100 \mathrm{MHz}, \mathrm{CDCl}_{3}\right): \delta 161.9,147.8,120.6,58.8,49.9,46.4$, 42.9, 33.2, 32.3, 27.0, 26.6, 25.2, 25.0, 19.7, 13.5. LRMS (ESI) $\mathrm{m} / \mathrm{z}$ (rel int): (pos) 322 ([M + H] $]^{+}, 100$ ), 282 $\left(\left[\mathrm{C}_{15} \mathrm{H}_{32} \mathrm{~N}_{5}\right]^{+}\right.$, 42). HRMS (ESI) $\mathrm{m} / \mathrm{z}$ calcd for C17H32N5O ${ }^{+}\left([\mathrm{M}+\mathrm{H}]^{+}\right) 322.2601$, found 322.2603.

(S)-4-Isopropyl-1-[4-(1-butyl-1,2,3-triazol-4-yl)butyl]imidazolidin-2-one (10c). 10c was synthesized according to the procedure described for the synthesis of 10a. Diamine $9 \mathrm{c}(2.05 \mathrm{~g}, 7.30 \mathrm{mmol})$, $\mathrm{Et}_{3} \mathrm{~N}(2.60 \mathrm{~mL}, 18.2 \mathrm{mmol})$ and CDI $(1.77 \mathrm{~g}, 10.9 \mathrm{mmol})$ were used. Reaction time: $14.5 \mathrm{~h}$. Yield: $1.78 \mathrm{~g}$, 79\%. White solid. TLC: $\mathrm{R}_{f} 0.29\left(30: 1 \mathrm{CH}_{2} \mathrm{Cl}_{2} / \mathrm{MeOH}(\times 2)\right) . \mathrm{mp}: 109.7-111.7^{\circ} \mathrm{C}$. $[\alpha]_{\mathrm{D}}^{21.7}=-9.0(\mathrm{c} 1.0$, $\mathrm{MeOH}) .{ }^{1} \mathrm{H}$ NMR $\left(400 \mathrm{MHz}, \mathrm{CDCl}_{3}\right): \delta 7.27(\mathrm{~s}, 1 \mathrm{H}), 4.32(\mathrm{~s}, 1 \mathrm{H}), 4.31(\mathrm{t}, J=7.2 \mathrm{~Hz}, 2 \mathrm{H}), 3.46(\mathrm{t}, J=8.4$ $\mathrm{Hz}, 1 \mathrm{H}), 3.36(\mathrm{ddd}, J=16.0,7.2,1.6 \mathrm{~Hz}, 1 \mathrm{H}), 3.26(\mathrm{dt}, J=13.6,7.2 \mathrm{~Hz}, 1 \mathrm{H}), 3.14(\mathrm{dt}, J=13.6,7.2 \mathrm{~Hz}, 1 \mathrm{H})$, $3.06(\mathrm{dd}, J=8.4,7.2 \mathrm{~Hz}, 1 \mathrm{H}), 2.75(\mathrm{t}, J=7.6 \mathrm{~Hz}, 2 \mathrm{H}), 1.87$ (quintet, $J=7.2 \mathrm{~Hz}, 2 \mathrm{H}), 1.73-1.63(\mathrm{~m}, 3 \mathrm{H})$, $1.60(\mathrm{~m}, 2 \mathrm{H}), 1.35$ (sextet, $J=7.2 \mathrm{~Hz}, 2 \mathrm{H}), 0.95(\mathrm{t}, J=7.2 \mathrm{~Hz}, 3 \mathrm{H}), 0.92(\mathrm{~d}, J=6.8 \mathrm{~Hz}, 3 \mathrm{H}), 0.88(\mathrm{~d}, J=6.8$ $\mathrm{Hz}, 3 \mathrm{H}) .{ }^{13} \mathrm{C}$ NMR $\left(100 \mathrm{MHz}, \mathrm{CDCl}_{3}\right): \delta 162.0,147.8,120.6,55.8,49.9,48.8,43.0,33.1,32.3$ 27.1, 26.6, 25.3, 19.7, 18.4, 17.9, 13.5. LRMS (ESI) $m / z$ (rel int): (pos) 308 ([M + H $\left.]^{+}, 100\right), 282\left(\left[\mathrm{C}_{15} \mathrm{H}_{32} \mathrm{~N}_{5}\right]^{+}, 25\right)$. HRMS (ESI) $\mathrm{m} / \mathrm{z}$ calcd for $\mathrm{C} 16 \mathrm{H} 30 \mathrm{~N}^{2} \mathrm{O}^{+}\left([\mathrm{M}+\mathrm{H}]^{+}\right) 308.2445$, found 308.2450.

(S)-4-[4-(4-Benzyl-2-oxoimidazolidin-1-yl)butyl]-1-butyl-3-methyl-1H-1,2,3-triazolium iodide (12a). In a screw-cap vial, a mixture of 10a (161 mg, $454 \mu \mathrm{mol})$ and MeI $(28.3 \mu \mathrm{L}, 454 \mu \mathrm{mol})$ was stirred at $80^{\circ} \mathrm{C}$. After $24 \mathrm{~h}$, the reaction mixture was cooled to rt. The purification by column chromatography on silica gel (15:1 $\left.\mathrm{CH}_{2} \mathrm{Cl}_{2} / \mathrm{MeOH}\right)$ afforded a desired compound $12 \mathrm{a}(183 \mathrm{mg}, 81 \%)$ as a light-yellow sticky gum. TLC: $\mathrm{R}_{f} 0.15\left(10: 1 \mathrm{CH}_{2} \mathrm{Cl}_{2} / \mathrm{MeOH}\right) \cdot[\alpha]_{\mathrm{D}}^{22.3}=-16.0$ (c 1.0, $\left.\mathrm{CHCl}_{3}\right) .{ }^{1} \mathrm{H} \mathrm{NMR}\left(400 \mathrm{MHz}, \mathrm{CDCl}_{3}\right): \delta 9.33(\mathrm{~s}$, $1 \mathrm{H}), 7.31(\mathrm{~m}, 2 \mathrm{H}), 7.25-7.19(\mathrm{~m}, 3 \mathrm{H}), 4.68(\mathrm{t}, J=7.6 \mathrm{~Hz}, 2 \mathrm{H}), 4.36(\mathrm{~s}, 1 \mathrm{H}), 4.28(\mathrm{~s}, 3 \mathrm{H}), 3.97(\mathrm{~m}, 1 \mathrm{H}), 3.62$ $(\mathrm{t}, J=8.8 \mathrm{~Hz}, 1 \mathrm{H}), 3.32(\mathrm{dt}, J=14.4,7.2 \mathrm{~Hz}, 1 \mathrm{H}), 3.18(\mathrm{dd}, J=8.8,6.4 \mathrm{~Hz}, 1 \mathrm{H}), 3.16(\mathrm{~m}, 1 \mathrm{H}), 3.04(\mathrm{~m}, 2 \mathrm{H})$, $2.87(\mathrm{dd}, J=13.6,5.6 \mathrm{~Hz}, 1 \mathrm{H}), 2.81(\mathrm{dd}, J=13.6,7.6 \mathrm{~Hz}, 1 \mathrm{H}), 2.03$ (quintet, $J=7.6 \mathrm{~Hz}, 2 \mathrm{H}), 1.84(\mathrm{~m}, 2 \mathrm{H})$, $1.70(\mathrm{~m}, 2 \mathrm{H}), 1.44$ (sextet, $J=7.6 \mathrm{~Hz}, 2 \mathrm{H}), 0.98(\mathrm{t}, J=7.6 \mathrm{~Hz}, 3 \mathrm{H}) .{ }^{13} \mathrm{C} \mathrm{NMR}\left(100 \mathrm{MHz}, \mathrm{CDCl}_{3}\right): \delta 162.1$, 144.5, 137.1, 129.6, 129.3, 128.8, 126.8, 54.0, 51.3, 50.2, 42.0, 41.8, 38.9, 31.3, 26.6, 24.0, 23.2, 19.5, 13.5 . LRMS (FAB) $m / z$ (rel int): (pos) $370\left(\left[\mathrm{C}_{21} \mathrm{H}_{32} \mathrm{~N}_{5} \mathrm{O}\right]^{+}, 100\right), 278\left(\left[\mathrm{C}_{21} \mathrm{H}_{32} \mathrm{~N}_{5} \mathrm{O}-\mathrm{Bn}\right]^{+}, 15\right)$. HRMS (FAB) $m / z$ calcd for $\mathrm{C}_{21} \mathrm{H}_{32} \mathrm{~N}_{5} \mathrm{O}^{+} 370.2601$, found 370.2603 .

(S)-4-[4-[4-(tert-Butyl)-2-oxoimidazolidin-1-yl])butyl]-1-butyl-3-methyl-1H-1,2,3-triazolium iodide (12b). 12b was synthesized according to the procedure described for the synthesis of 12a. 10b $(1.46 \mathrm{~g}, 4.54 \mathrm{mmol})$ 
and MeI ( $290 \mu \mathrm{L}, 4.54 \mathrm{mmol})$ were used. Reaction time: $24 \mathrm{~h}$. Yield: $1.80 \mathrm{~g}, 86 \%$. Yellow gum. TLC: $\mathrm{R}_{f}$ $0.47\left(10: 1 \mathrm{CH}_{2} \mathrm{Cl}_{2} / \mathrm{MeOH}\right) .[\alpha]_{\mathrm{D}}^{22.8}=-8.1\left(\mathrm{c} 1.0, \mathrm{CHCl}_{3}\right) .{ }^{1} \mathrm{H} \mathrm{NMR}\left(400 \mathrm{MHz}, \mathrm{CDCl}_{3}\right): \delta 9.41(\mathrm{~s}, 1 \mathrm{H})$, $4.70(\mathrm{t}, J=7.6 \mathrm{~Hz}, 2 \mathrm{H}), 4.53(\mathrm{br} \mathrm{s}, 1 \mathrm{H}), 4.26(\mathrm{~s}, 3 \mathrm{H}), 3.51-3.45(\mathrm{~m}, 2 \mathrm{H}), 3.36(\mathrm{dt}, J=14.4,6.8 \mathrm{~Hz}, 1 \mathrm{H})$, $3.22-3.14(\mathrm{~m}, 2 \mathrm{H}), 3.04(\mathrm{td}, J=8.0,2.8 \mathrm{~Hz}, 2 \mathrm{H}), 2.04$ (quintet, $J=7.6 \mathrm{~Hz}, 2 \mathrm{H}), 1.86$ (quintet, $J=7.6 \mathrm{~Hz}$, $2 \mathrm{H}), 1.73$ (sextet, $J=7.6 \mathrm{~Hz}, 2 \mathrm{H}), 1.44$ (sextet, $J=7.6 \mathrm{~Hz}, 2 \mathrm{H}), 0.99(\mathrm{t}, J=7.6 \mathrm{~Hz}, 3 \mathrm{H}), 0.88(\mathrm{~s}, 9 \mathrm{H}) .{ }^{13} \mathrm{C}$ NMR (100 MHz, $\left.\mathrm{CDCl}_{3}\right): \delta 162.2,144.5,129.7,58.8,54.0,46.5,41.7,38.5,33.3,31.3,26.4,25.1,24.0,22.9$, 19.5, 13.4. LRMS (FAB) $m / z$ (rel int): (pos) $336\left(\left[\mathrm{C}_{18} \mathrm{H}_{34} \mathrm{~N}_{5} \mathrm{O}\right]^{+}, 100\right), 278\left(\left[\mathrm{C}_{18} \mathrm{H}_{34} \mathrm{~N}_{5} \mathrm{O}-t \text {-Bu }\right]^{+}, 15\right)$. HRMS (FAB) $m / z$ calcd for $\mathrm{C}_{18} \mathrm{H}_{34} \mathrm{~N}_{5} \mathrm{O}^{+} 336.2758$, found 336.2758 .

(S)-4-[4-(4-Isopropyl-2-oxoimidazolidin-1-yl)butyl]-1-butyl-3-methyl-1H-1,2,3-triazolium iodide (12c). 12c was synthesized according to the procedure described for the synthesis of 12a. 10c $(1.78 \mathrm{~g}, 5.78 \mathrm{mmol})$ and MeI $(360 \mu \mathrm{L}, 5.78 \mathrm{mmol})$ were used. Reaction time: $24 \mathrm{~h}$. Yield: $2.39 \mathrm{~g}, 92 \%$. Colorless oil. TLC: $\mathrm{R}_{f}$ $0.26\left(10: 1 \mathrm{CH}_{2} \mathrm{Cl}_{2} / \mathrm{MeOH}\right) \cdot[\alpha]_{\mathrm{D}}^{21.8}=-6.0(\mathrm{c} 0.50, \mathrm{MeOH}) .{ }^{1} \mathrm{H} \mathrm{NMR}\left(400 \mathrm{MHz}, \mathrm{CDCl}_{3}\right): \delta 9.39(\mathrm{~s}, 1 \mathrm{H})$, $4.69(\mathrm{t}, J=7.6 \mathrm{~Hz}, 2 \mathrm{H}), 4.52(\mathrm{br} \mathrm{s}, 1 \mathrm{H}), 4.27(\mathrm{~s}, 3 \mathrm{H}), 3.56(\mathrm{t}, J=8.8 \mathrm{~Hz}, 1 \mathrm{H}), 3.46(\mathrm{qm}, J=8.8 \mathrm{~Hz}, 1 \mathrm{H}), 3.36$ $(\mathrm{dt}, J=14.4,7.2 \mathrm{~Hz}, 1 \mathrm{H}), 3.19(\mathrm{dt}, J=14.4,6.0 \mathrm{~Hz}, 1 \mathrm{H}), 3.11(\mathrm{dd}, J=8.8,7.6 \mathrm{~Hz}, 1 \mathrm{H}), 3.04(\mathrm{~m}, 2 \mathrm{H}), 2.04$ (quintet, $J=7.6 \mathrm{~Hz}, 2 \mathrm{H}), 1.86(\mathrm{~m}, 2 \mathrm{H}), 1.73(\mathrm{~m}, 1 \mathrm{H}), 1.68$ (sextet, $J=7.6 \mathrm{~Hz}, 2 \mathrm{H}), 1.44$ (sextet, $J=7.6 \mathrm{~Hz}$, $2 \mathrm{H}), 0.99(\mathrm{t}, J=7.6 \mathrm{~Hz}, 3 \mathrm{H}), 0.93(\mathrm{~d}, J=6.8 \mathrm{~Hz}, 3 \mathrm{H}), 0.89(\mathrm{~d}, J=6.8 \mathrm{~Hz}, 3 \mathrm{H}) .{ }^{13} \mathrm{C} \mathrm{NMR}(100 \mathrm{MHz}$, $\left.\mathrm{CDCl}_{3}\right): \delta 162.5,144.6,129.8,55.9,54.0,48.9,41.8,38.6,33.1,31.3,26.4,24.0,23.1,19.5,18.5,17.9,13.4$. LRMS (FAB) $m / z$ (rel int): (pos) $322\left(\left[\mathrm{C}_{18} \mathrm{H}_{34} \mathrm{~N}_{5} \mathrm{O}\right]^{+}, 100\right), 278\left(\left[\mathrm{C}_{18} \mathrm{H}_{34} \mathrm{~N}_{5} \mathrm{O}-i \text {-Pr] }\right]^{+}, 10\right)$. HRMS (FAB) $\mathrm{m} / \mathrm{z}$ calcd for $\mathrm{C}_{18} \mathrm{H}_{34} \mathrm{~N}_{5} \mathrm{O}^{+} 322.2601$, found 322.2603.

(S)-4-[4-(4-Benzyl-2-oxoimidazolidin-1-yl)butyl]-1-butyl-3-methyl-1H-1,2,3-triazolium

bis(trifluoromethanesulfonyl)imide (1a). In a $25 \mathrm{~mL}$ roundbottom flask, 12a (463 mg, $930 \mu \mathrm{mol})$ and $\operatorname{LiNTf}_{2}(267 \mathrm{mg}, 930 \mu \mathrm{mol})$ were dissolved in deionized water $(1.6 \mathrm{~mL})$. The reaction mixture was stirred at $40{ }^{\circ} \mathrm{C}$ for $24 \mathrm{~h}$. Upon completion of the reaction, the reaction mixture was cooled to rt, and extracted with $\mathrm{CH}_{2} \mathrm{Cl}_{2}(5 \times 9 \mathrm{~mL})$. The combined organic extracts were dried over anhydrous $\mathrm{Na}_{2} \mathrm{SO}_{4}$, and filtered. The concentration by rotary evaporation afforded a desired $\mathbf{1 a}(577 \mathrm{mg}, 95 \%)$ as a light yellow liquid. TLC: $\mathrm{R}_{f} 0.10\left(10: 1 \mathrm{CH}_{2} \mathrm{Cl}_{2} / \mathrm{MeOH}\right) \cdot[\alpha]_{\mathrm{D}}^{22.4}=-6.5\left(\mathrm{c} 1.0, \mathrm{CHCl}_{3}\right) .{ }^{1} \mathrm{H} \mathrm{NMR}$ $\left(400 \mathrm{MHz}, \mathrm{CDCl}_{3}\right): \delta 8.40(\mathrm{~s}, 1 \mathrm{H}), 7.31(\mathrm{~m}, 2 \mathrm{H}), 7.26-7.17(\mathrm{~m}, 3 \mathrm{H}), 4.53(\mathrm{t}, J=7.6 \mathrm{~Hz}, 2 \mathrm{H}), 4.34(\mathrm{br} \mathrm{s}, 1 \mathrm{H})$, $4.21(\mathrm{~s}, 3 \mathrm{H}), 3.94(\mathrm{~m}, 1 \mathrm{H}), 3.57(\mathrm{t}, J=8.8 \mathrm{~Hz}, 1 \mathrm{H}), 3.34(\mathrm{dt}, J=14.4,6.4 \mathrm{~Hz}, 1 \mathrm{H}), 3.18(\mathrm{dt}, J=14.4,6.0$ $\mathrm{Hz}, 1 \mathrm{H}), 3.17(\mathrm{dd}, J=8.8,6.4 \mathrm{~Hz}, 1 \mathrm{H}), 2.93(\mathrm{~m}, 2 \mathrm{H}), 2.87(\mathrm{dd}, J=13.6,5.6 \mathrm{~Hz}, 1 \mathrm{H}), 2.77(\mathrm{dd}, J=13.6$, $8.0 \mathrm{~Hz}, 1 \mathrm{H}), 1.99$ (quintet, $J=7.6 \mathrm{~Hz}, 2 \mathrm{H}), 1.77(\mathrm{~m}, 2 \mathrm{H}), 1.69(\mathrm{~m}, 2 \mathrm{H}), 1.41$ (sextet, $J=7.6 \mathrm{~Hz}, 2 \mathrm{H}), 0.99(\mathrm{t}$, $J=7.6 \mathrm{~Hz}, 3 \mathrm{H}) .{ }^{13} \mathrm{C}$ NMR $\left(100 \mathrm{MHz}\right.$, acetone- $\left.d_{6}\right): \delta 162.4,145.9,138.6,130.2,129.4,129.1,127.4,121.1(\mathrm{q}$, $\left.J_{\mathrm{C}-\mathrm{F}}=319.6 \mathrm{~Hz}\right), 54.4,51.9,50.4,42.6,42.5,38.1,32.0,27.2,24.7,23.1,20.0,13.6 .{ }^{19} \mathrm{~F}$ NMR $(376 \mathrm{MHz}$, $\left.\mathrm{CDCl}_{3}\right): \delta$-79.3. LRMS (FAB) $\mathrm{m} / z$ (rel int): (pos) $370\left(\left[\mathrm{C}_{21} \mathrm{H}_{32} \mathrm{~N}_{5} \mathrm{O}\right]^{+}, 100\right), 278\left(\left[\mathrm{C}_{21} \mathrm{H}_{32} \mathrm{~N}_{5} \mathrm{O}-\mathrm{Bn}\right]^{+}\right.$, 25). HRMS (FAB) $m / z$ calcd for $\mathrm{C}_{21} \mathrm{H}_{32} \mathrm{~N}_{5} \mathrm{O}^{+} 370.2601$, found 370.2602 .

(S)-4-[4-[4-(tert-Butyl)-2-oxoimidazolidin-1-yl]butyl]-1-butyl-3-methyl-1H-1,2,3-triazolium bis(trifluoromethanesulfonyl)imide ( $\mathbf{1 b})$. $\mathbf{1} \mathbf{b}$ was synthesized according to the procedure described for the synthesis of 1a. 12b (331 mg, $714 \mathrm{mmol})$ and $\operatorname{LiNTf}_{2}(205 \mathrm{mg}, 714 \mathrm{mmol})$ were used. Reaction time: 24 h. Yield: $417 \mathrm{mg}, 95 \%$. Colorless oil. TLC: $\mathrm{R}_{\mathrm{f}} 0.56\left(10: 1 \mathrm{CH}_{2} \mathrm{Cl}_{2} / \mathrm{MeOH}\right) .[\alpha]_{\mathrm{D}}^{22.9}=-5.7$ (c 1.0, $\left.\mathrm{CHCl}_{3}\right)$. ${ }^{1} \mathrm{H} \mathrm{NMR}\left(400 \mathrm{MHz}, \mathrm{CDCl}_{3}\right): \delta 8.45(\mathrm{~s}, 1 \mathrm{H}), 4.54(\mathrm{t}, J=7.6 \mathrm{~Hz}, 2 \mathrm{H}), 4.34(\mathrm{~s}, 1 \mathrm{H}), 4.22(\mathrm{~s}, 3 \mathrm{H}), 3.45-3.41$ $(\mathrm{m}, 2 \mathrm{H}), 3.34(\mathrm{dt}, J=14.4,6.4 \mathrm{~Hz}, 1 \mathrm{H}), 3.20(\mathrm{t}, J=6.0 \mathrm{~Hz}, 1 \mathrm{H}), 3.16(\mathrm{~m}, 1 \mathrm{H}), 2.96(\mathrm{~m}, 2 \mathrm{H}), 1.99$ (quintet, $J=7.6 \mathrm{~Hz}, 2 \mathrm{H}), 1.79$ (quintet, $J=7.6 \mathrm{~Hz}, 2 \mathrm{H}), 1.71(\mathrm{~m}, 2 \mathrm{H}), 1.42$ (sextet, $J=7.6 \mathrm{~Hz}, 2 \mathrm{H}), 0.99(\mathrm{t}, J=7.6$ $\mathrm{Hz}, 3 \mathrm{H}), 0.87$ (s, 9H). ${ }^{13} \mathrm{C} \mathrm{NMR}\left(100 \mathrm{MHz}, \mathrm{CDCl}_{3}\right): \delta 162.2,144.8,128.3,119.8$ (q, JC-F $\left.=319.7 \mathrm{~Hz}\right), 58.8$, 53.9, 46.3, 41.6, 37.4, 33.2, 31.0, 26.3, 24.9, 23.7, 22.3, 19.4, 13.2. $\left.{ }^{19} \mathrm{~F} \mathrm{NMR} \mathrm{(376} \mathrm{MHz,} \mathrm{CDCl}_{3}\right): \delta-79.2$. LRMS (FAB) $m / z$ (rel int): (pos) $336\left(\left[\mathrm{C}_{18} \mathrm{H}_{34} \mathrm{~N}_{5} \mathrm{O}\right]^{+}, 100\right), 278\left(\left[\mathrm{C}_{18} \mathrm{H}_{34} \mathrm{~N}_{5} \mathrm{O}-t \text {-Bu }\right]^{+}, 13\right)$. HRMS (FAB) $m / z$ calcd for $\mathrm{C}_{18} \mathrm{H}_{34} \mathrm{~N}_{5} \mathrm{O}^{+} 336.2758$, found 336.2760 .

(S)-4-[4-(4-Isopropyl-2-oxoimidazolidin-1-yl)butyl]-1-butyl-3-methyl-1H-1,2,3-triazolium bis(trifluoromethanesulfonyl)imide (1c). 1c was synthesized according to the procedure described for the synthesis of 1a. 12c (1.92 g, $4.27 \mathrm{mmol})$ and $\mathrm{LiNTf}_{2}(1.23 \mathrm{~g}, 4.27 \mathrm{mmol})$ were used. Reaction time: 
24 h. Yield: 2.46 g, $96 \%$. Colorless oil. TLC: $\mathrm{R}_{f} 0.39\left(10: 1 \mathrm{CH}_{2} \mathrm{Cl}_{2} / \mathrm{MeOH}\right) \cdot[\alpha]_{\mathrm{D}}^{21.7}=-3.9$ (c 1.0, $\left.\mathrm{MeOH}\right)$. ${ }^{1} \mathrm{H}$ NMR $\left(400 \mathrm{MHz}, \mathrm{CDCl}_{3}\right): \delta 8.34(\mathrm{~s}, 1 \mathrm{H}), 4.52(\mathrm{t}, J=7.6 \mathrm{~Hz}, 2 \mathrm{H}), 4.46(\mathrm{~s}, 1 \mathrm{H}), 4.21(\mathrm{~s}, 3 \mathrm{H}), 3.52(\mathrm{t}$, $J=8.8 \mathrm{~Hz}, 1 \mathrm{H}), 3.41(\mathrm{qd}, J=8.8,1.6 \mathrm{~Hz}, 1 \mathrm{H}), 3.33(\mathrm{dt}, J=14.4,6.4 \mathrm{~Hz}, 1 \mathrm{H}), 3.17(\mathrm{dt}, J=14.4,6.4 \mathrm{~Hz}, 1 \mathrm{H})$, $3.09(\mathrm{dd}, J=8.8,7.6 \mathrm{~Hz}, 1 \mathrm{H}), 2.94(\mathrm{~m}, 2 \mathrm{H}), 1.98$ (quintet, $J=7.6 \mathrm{~Hz}, 2 \mathrm{H}), 1.77$ (quintet, $J=7.6 \mathrm{~Hz}, 2 \mathrm{H}$ ), $1.73-1.64(\mathrm{~m}, 3 \mathrm{H}), 1.41$ (sextet, $J=7.6 \mathrm{~Hz}, 2 \mathrm{H}), 0.99(\mathrm{t}, J=7.6 \mathrm{~Hz}, 3 \mathrm{H}), 0.92(\mathrm{~d}, J=6.8 \mathrm{~Hz}, 3 \mathrm{H}), 0.88(\mathrm{~d}$, $J=6.8 \mathrm{~Hz}, 3 \mathrm{H}) .{ }^{13} \mathrm{C}$ NMR $\left(100 \mathrm{MHz}, \mathrm{CDCl}_{3}\right): \delta 162.4,144.8,128.2,119.8\left(\mathrm{q}, J_{\mathrm{C}-\mathrm{F}}=319.7 \mathrm{~Hz}\right), 55.9,53.9$, $48.7,41.7,37.4,33.0,31.0,26.3,23.6,22.4,19.4,18.2,17.7,13.2 .{ }^{19} \mathrm{~F} \mathrm{NMR}\left(376 \mathrm{MHz}, \mathrm{CDCl}_{3}\right): \delta-79.3$. LRMS (FAB) $m / z$ (rel int): (pos) $322\left(\left[\mathrm{C}_{17} \mathrm{H}_{32} \mathrm{~N}_{5} \mathrm{O}\right]^{+}, 100\right), 278\left(\left[\mathrm{C}_{1} \mathrm{H}_{34} \mathrm{~N}_{5} \mathrm{O}-i-\mathrm{Pr}\right]^{+}, 11\right), 57\left(\left[\mathrm{C}_{4} \mathrm{H}_{9}\right]^{+}\right.$, 62). HRMS (FAB) $m / z$ calcd for $\mathrm{C}_{18} \mathrm{H}_{34} \mathrm{~N}_{5} \mathrm{O}^{+} 322.2601$, found 322.2607 .

(S)-4-[4-(4-Benzyl-2-oxoimidazolidin-1-yl)butyl]-1,3-dibutyl-1H-1,2,3-triazolium iodide (16a). In a screw-cap vial, a mixture of 10a (354 mg, $995 \mu \mathrm{mol})$ and $n$-BuI $(115 \mu \mathrm{L}, 995 \mu \mathrm{mol})$ in DMF $(100 \mu \mathrm{L})$ was stirred at $100{ }^{\circ} \mathrm{C}$. After $24 \mathrm{~h}$, the reaction mixture was cooled to rt. The purification by column chromatography on silica gel (30:1 $\left.\mathrm{CH}_{2} \mathrm{Cl}_{2} / \mathrm{MeOH}\right)$ afforded a desired triazolium iodide $16 \mathrm{a}$ (467 $\mathrm{mg}$, $92 \%$ ) as a yellow oil. TLC: $\mathrm{R}_{f} 0.36\left(20: 1 \mathrm{CH}_{2} \mathrm{Cl}_{2} / \mathrm{MeOH}\right) \cdot[\alpha]_{\mathrm{D}}^{20.0}=-37.2$ (c $\left.1.2, \mathrm{CHCl}_{3}\right) .{ }^{1} \mathrm{H} \mathrm{NMR}\left(400 \mathrm{MHz}, \mathrm{CDCl}_{3}\right): \delta$ $9.60(\mathrm{~s}, 1 \mathrm{H}), 7.33-7.30(\mathrm{~m}, 2 \mathrm{H}), 7.26-7.19(\mathrm{~m}, 3 \mathrm{H}), 4.73(\mathrm{t}, J=7.2 \mathrm{~Hz}, 2 \mathrm{H}), 4.48(\mathrm{t}, J=7.2 \mathrm{~Hz}, 2 \mathrm{H}), 4.31(\mathrm{br}$ $\mathrm{s}, 1 \mathrm{H}), 3.97(\mathrm{~m}, 1 \mathrm{H}), 3.64(\mathrm{t}, J=8.8 \mathrm{~Hz}, 1 \mathrm{H}), 3.35(\mathrm{dt}, J=14.4,6.8 \mathrm{~Hz}, 1 \mathrm{H}), 3.22-3.14(\mathrm{~m}, 2 \mathrm{H}), 2.98(\mathrm{~m}$, $2 \mathrm{H}), 2.90(\mathrm{dd}, J=13.2,5.2 \mathrm{~Hz}, 1 \mathrm{H}), 2.77(\mathrm{dd}, J=13.2,8.4 \mathrm{~Hz}, 1 \mathrm{H}), 2.04$ (quintet, $J=7.6 \mathrm{~Hz}, 2 \mathrm{H}), 1.95(\mathrm{~m}$, $2 \mathrm{H}), 1.86$ (quintet, $J=7.2 \mathrm{~Hz}, 2 \mathrm{H}), 1.74(\mathrm{~m}, 2 \mathrm{H}), 1.42$ (sextet, $J=7.2 \mathrm{~Hz}, 4 \mathrm{H}), 1.01(\mathrm{t}, J=7.2 \mathrm{~Hz}, 3 \mathrm{H}), 0.99$ $(\mathrm{t}, J=7.2 \mathrm{~Hz}, 3 \mathrm{H}) .{ }^{13} \mathrm{C}$ NMR $\left(100 \mathrm{MHz}, \mathrm{CDCl}_{3}\right): \delta 161.8,143.9,137.1,130.1,129.1,128.8,126.9,54.1$, 51.4, 51.2, 50.4, 42.0, 41.6, 31.4, 31.0, 26.4, 24.2, 22.7, 19.6, 19.5, 13.4, 13.3. LRMS (ESI) $m / z$ (rel int): (pos) $412\left(\left[\mathrm{C} 24 \mathrm{H} 38 \mathrm{~N}^{2} \mathrm{O}^{+}, 100\right), 394\left(\left[\mathrm{C} 24 \mathrm{H} 38 \mathrm{~N} 5 \mathrm{O}-\mathrm{H}_{2} \mathrm{O}\right]^{+}, 3\right)\right.$. HRMS (ESI) $\mathrm{m} / \mathrm{z}$ calcd for C24H38N5O ${ }^{+}$ 412.3071, found 412.3071 .

(S)-4-[4-(4-(tert-Butyl)-2-oxoimidazolidin-1-yl)butyl]-1,3-dibutyl-1H-1,2,3-triazolium iodide (16b). 16b was synthesized according to the procedure described for the synthesis of $\mathbf{1 6 a} .10 \mathbf{b}(161 \mathrm{mg}, 500 \mu \mathrm{mol})$ and $n$-BuI $(58.0 \mu \mathrm{L}, 500 \mu \mathrm{mol})$ in DMF (50 $\mu \mathrm{L})$ were used. Reaction time: $38 \mathrm{~h}$. Yield: $229 \mathrm{mg}, 91 \%$. Yellow oil. TLC: $\mathrm{R}_{f} 0.16\left(20: 1 \mathrm{CH}_{2} \mathrm{Cl}_{2} / \mathrm{MeOH}\right) \cdot[\alpha]_{\mathrm{D}}^{20.0}=-38.4$ (c $\left.0.82, \mathrm{CHCl}_{3}\right) .{ }^{1} \mathrm{H} \mathrm{NMR}\left(400 \mathrm{MHz}, \mathrm{CDCl}_{3}\right)$ : $\delta 9.63(\mathrm{~s}, 1 \mathrm{H}), 4.74(\mathrm{t}, J=7.2 \mathrm{~Hz}, 2 \mathrm{H}), 4.48(\mathrm{t}, J=7.2 \mathrm{~Hz}, 2 \mathrm{H}), 4.34(\mathrm{br} \mathrm{s}, 1 \mathrm{H}), 3.51-3.45(\mathrm{~m}, 2 \mathrm{H}), 3.36$ $(\mathrm{m}, 1 \mathrm{H}), 3.21-3.14(\mathrm{~m}, 2 \mathrm{H}), 3.00(\mathrm{td}, J=8.0,5.2 \mathrm{~Hz}, 2 \mathrm{H}), 2.04$ (quintet, $J=7.2 \mathrm{~Hz}, 2 \mathrm{H}), 1.95$ (quintet, $J=7.2 \mathrm{~Hz}, 2 \mathrm{H}$ ), 1.87 (quintet, $J=7.2 \mathrm{~Hz}, 2 \mathrm{H}$ ), 1.74 (sextet, $J=7.6 \mathrm{~Hz}, 2 \mathrm{H}$ ), 1.43 (sextet, $J=7.2 \mathrm{~Hz}, 2 \mathrm{H}$ ), 1.42 (sextet, $J=7.2 \mathrm{~Hz}, 2 \mathrm{H}), 1.01(\mathrm{t}, J=7.2 \mathrm{~Hz}, 3 \mathrm{H}), 0.99(\mathrm{t}, J=7.2 \mathrm{~Hz}, 3 \mathrm{H}), 0.88(\mathrm{~s}, 9 \mathrm{H}) .{ }^{13} \mathrm{C}$ NMR $(100$ $\left.\mathrm{MHz}, \mathrm{CDCl}_{3}\right): \delta 162.1,143.9,130.1,58.8,54.1,51.1,46.5,41.5,33.3,31.4,31.0,26.3,25.1,24.2,22.6,19.6$, 19.5, 13.4, 13.3. LRMS (ESI) $\mathrm{m} / z$ (rel int): (pos) $378\left(\left[\mathrm{C} 21 \mathrm{H} 40 \mathrm{~N}^{2} \mathrm{O}\right]^{+}, 100\right), 361\left(\left[\mathrm{C} 21 \mathrm{H}_{39} \mathrm{~N} 5 \mathrm{O}-\mathrm{H}_{2} \mathrm{O}\right]^{+}\right.$, 2). HRMS (ESI) $\mathrm{m} / \mathrm{z}$ calcd for $\mathrm{C} 21 \mathrm{H} 40 \mathrm{~N}^{2} \mathrm{O}^{+} 378.3227$, found 378.3227 .

(S)-4-[4-(4-Isopropyl-2-oxoimidazolidin-1-yl)butyl]-1,3-dibutyl-1H-1,2,3-triazolium iodide (16c). 16c was synthesized according to the procedure described for the synthesis of 16a. 10c $(154 \mathrm{mg}, 500 \mu \mathrm{mol})$ and $n$-BuI $(58 \mu \mathrm{L}, 500 \mu \mathrm{mol})$ in DMF ( $50 \mu \mathrm{L})$ were used. Reaction time: $34 \mathrm{~h}$. Yield: $190 \mathrm{mg}, 77 \%$. Yellow oil. TLC: $\mathrm{R}_{f} 0.2\left(10: 1 \mathrm{CH}_{2} \mathrm{Cl}_{2} / \mathrm{MeOH}\right) .[\alpha]_{\mathrm{D}}^{20.0}=-39.8\left(\mathrm{c} 0.54, \mathrm{CHCl}_{3}\right) .{ }^{1} \mathrm{H} \mathrm{NMR}\left(400 \mathrm{MHz}, \mathrm{CDCl}_{3}\right): \delta 9.64(\mathrm{~s}$, $1 \mathrm{H}), 4.74(\mathrm{t}, J=7.2 \mathrm{~Hz}, 2 \mathrm{H}), 4.48(\mathrm{t}, J=7.2 \mathrm{~Hz}, 2 \mathrm{H}), 4.35(\mathrm{br} \mathrm{s}, 1 \mathrm{H}), 3.58(\mathrm{t}, J=8.8 \mathrm{~Hz}, 1 \mathrm{H}), 3.45(\mathrm{qm}$, $J=7.2 \mathrm{~Hz}, 1 \mathrm{H}), 3.35(\mathrm{dt}, J=14.4,2.8 \mathrm{~Hz}, 1 \mathrm{H}), 3.18(\mathrm{dt}, J=14.4,6.4 \mathrm{~Hz}, 1 \mathrm{H}), 3.11(\mathrm{dd}, J=8.8,7.6 \mathrm{~Hz}, 1 \mathrm{H})$, $2.99(\mathrm{~m}, 2 \mathrm{H}), 2.04$ (quintet, $J=7.6 \mathrm{~Hz}, 2 \mathrm{H}), 1.95$ (quintet, $J=7.2 \mathrm{~Hz}, 2 \mathrm{H}), 1.87(\mathrm{~m}, 2 \mathrm{H}), 1.78-1.65(\mathrm{~m}$, $3 \mathrm{H}), 1.42$ (sextet, $J=7.6 \mathrm{~Hz}, 4 \mathrm{H}), 1.01(\mathrm{t}, J=7.2 \mathrm{~Hz}, 3 \mathrm{H}), 0.99(\mathrm{t}, J=7.2 \mathrm{~Hz}, 3 \mathrm{H}), 0.93(\mathrm{~d}, J=6.8 \mathrm{~Hz}$, $3 \mathrm{H}), 0.90(\mathrm{~d}, J=6.8 \mathrm{~Hz}, 3 \mathrm{H}) .{ }^{13} \mathrm{C}$ NMR $\left(100 \mathrm{MHz}, \mathrm{CDCl}_{3}\right): \delta 162.4,144.1,130.2,56.0,54.2,51.3,49.1$, 41.7, 33.2, 31.5, 31.1, 26.5, 24.4, 22.8, 19.7, 19.6, 18.5, 18.1, 13.6, 13.5. LRMS (ESI) $\mathrm{m} / \mathrm{z}$ (rel int): (pos) 364 $\left([\mathrm{C} 20 \mathrm{H} 38 \mathrm{~N} 5 \mathrm{O}]^{+}, 100\right), 387\left([\mathrm{C} 20 \mathrm{H} 38 \mathrm{~N} 5 \mathrm{O}+\mathrm{Na}]^{+}, 1\right) . \mathrm{HRMS}(\mathrm{ESI}) \mathrm{m} / \mathrm{z}$ calcd for $\mathrm{C} 20 \mathrm{H} 38 \mathrm{~N} 5 \mathrm{O}^{+} 364.3071$, found 364.3062 .

(S)-4-[4-(4-Benzyl-2-oxoimidazolidin-1-yl)butyl]-1,3-dibutyl-1H-1,2,3-triazolium bis(trifluoromethanesulfonyl)imide (2a). In a screw-cap vial, 16a (164 mg, $321 \mu \mathrm{mol})$ and $\mathrm{LiNTf}_{2}(92.2 \mathrm{mg}$, $321 \mu \mathrm{mol})$ were dissolved in deionized water $(0.54 \mathrm{~mL})$. The reaction mixture was stirred at $40{ }^{\circ} \mathrm{C}$ 
for $24 \mathrm{~h}$. Upon completion of the reaction, the reaction mixture was cooled to rt, and extracted with $\mathrm{CH}_{2} \mathrm{Cl}_{2}(3 \times 3 \mathrm{~mL})$. The combined organic extracts were dried over anhydrous $\mathrm{MgSO}_{4}$, and filtered. The concentration by rotary evaporation afforded the desired $\mathrm{NTf}_{2}$ salt $\mathbf{2 a}(192 \mathrm{mg}, 86 \%)$ as a yellow oil. TLC: $\mathrm{R}_{f} 0.53\left(15: 1 \mathrm{CH}_{2} \mathrm{Cl}_{2} / \mathrm{MeOH}\right) \cdot[\alpha]_{\mathrm{D}}^{20.0}=-42.1$ (c 1.1, $\left.\mathrm{CHCl}_{3}\right) .{ }^{1} \mathrm{H} \mathrm{NMR}\left(400 \mathrm{MHz}, \mathrm{CDCl}_{3}\right): \delta 8.43(\mathrm{~s}$, $1 \mathrm{H}), 7.33-7.30(\mathrm{~m}, 2 \mathrm{H}), 7.26-7.17(\mathrm{~m}, 3 \mathrm{H}), 4.54(\mathrm{t}, \mathrm{J}=7.6 \mathrm{~Hz}, 2 \mathrm{H}), 4.45(\mathrm{t}, J=7.6 \mathrm{~Hz}, 2 \mathrm{H}), 3.96(\mathrm{~m}, 1 \mathrm{H})$, $3.58(\mathrm{t}, J=8.8 \mathrm{~Hz}, 1 \mathrm{H}), 3.34(\mathrm{dt}, J=14.4,6.8 \mathrm{~Hz}, 1 \mathrm{H}), 3.22-3.14(\mathrm{~m}, 2 \mathrm{H}), 2.92(\mathrm{~m}, 2 \mathrm{H}), 2.87(\mathrm{dd}, J=13.6$, $5.2 \mathrm{~Hz}, 1 \mathrm{H}), 2.77(\mathrm{dd}, J=13.6,8.4 \mathrm{~Hz}, 1 \mathrm{H}), 1.99$ (quintet, $J=7.6 \mathrm{~Hz}, 2 \mathrm{H}$ ), 1.95 (quintet, $J=7.6 \mathrm{~Hz}, 2 \mathrm{H}$ ), $1.78(\mathrm{~m}, 2 \mathrm{H}), 1.69(\mathrm{~m}, 2 \mathrm{H}), 1.42$ (sextet, $J=7.6 \mathrm{~Hz}, 2 \mathrm{H}), 1.40$ (sextet, $J=7.6 \mathrm{~Hz}, 2 \mathrm{H}), 1.00(\mathrm{t}, J=7.6 \mathrm{~Hz}$, $3 \mathrm{H}), 0.99(\mathrm{t}, \mathrm{J}=7.6 \mathrm{~Hz}, 3 \mathrm{H}) .{ }^{13} \mathrm{C} \mathrm{NMR}\left(100 \mathrm{MHz}, \mathrm{CDCl}_{3}\right): \delta 161.9,144.3,137.0,129.1,128.8,128.6,126.9$, $119.8\left(\mathrm{q}, J_{\mathrm{C}-\mathrm{F}}=319.7 \mathrm{~Hz}\right), 54.0,51.4,50.9,50.2,41.9,41.6,31.1,30.8,26.3,23.9,22.3,19.5,19.4,13.3,13.2$. ${ }^{19} \mathrm{~F}$ NMR (376 MHz, $\left.\mathrm{CDCl}_{3}\right): \delta$-79.2. LRMS (ESI) $\mathrm{m} / z$ (rel int): (pos) 412 ([C24H38N5O] $\left.{ }^{+}, 100\right), 394$ $\left(\left[\mathrm{C} 24 \mathrm{H} 38 \mathrm{~N} 5 \mathrm{O}-\mathrm{H}_{2} \mathrm{O}\right]^{+}, 10\right)$. HRMS (ESI) m/z calcd for C24H38N5O ${ }^{+} 412.3071$, found 412.3069.

(S)-4-[4-(4-(tert-Butyl)-2-oxoimidazolidin-1-yl)butyl]-1,3-dibutyl-1H-1,2,3-triazolium

bis(trifluoromethanesulfonyl)imide (2b). $\mathbf{2 b}$ was synthesized according to the procedure described for the synthesis of 2a. 16b (199 mg, $393 \mu \mathrm{mol})$ and LiNTf $_{2}(113 \mathrm{mg}, 393 \mu \mathrm{mol})$ were used. Reaction time: $24 \mathrm{~h}$. Yield: $204 \mathrm{mg}, 79 \%$. Light yellow oil. TLC: $\mathrm{R}_{f} 0.69\left(10: 1 \mathrm{CH}_{2} \mathrm{Cl}_{2} / \mathrm{MeOH}(\times 2)\right)$. $[\alpha]_{\mathrm{D}}^{20.0}=-68.0(\mathrm{c} 0.40$, $\left.\mathrm{CHCl}_{3}\right) .{ }^{1} \mathrm{H} \mathrm{NMR}\left(400 \mathrm{MHz}, \mathrm{CDCl}_{3}\right): \delta 8.43(\mathrm{~s}, 1 \mathrm{H}), 4.54(\mathrm{t}, J=7.6 \mathrm{~Hz}, 2 \mathrm{H}), 4.46(\mathrm{t}, J=7.6 \mathrm{~Hz}, 2 \mathrm{H})$, 3.52-3.46 (m, 2H), $3.37(\mathrm{~m}, 1 \mathrm{H}), 3.22-3.14(\mathrm{~m}, 2 \mathrm{H}), 2.92(\mathrm{td}, J=8.0,4.8 \mathrm{~Hz}, 2 \mathrm{H}), 1.99$ (quintet, $J=7.6 \mathrm{~Hz}$, 2H), 1.95 (quintet, $J=7.6 \mathrm{~Hz}, 2 \mathrm{H}), 1.79$ (sextet, $J=7.6 \mathrm{~Hz}, 2 \mathrm{H}), 1.71(\mathrm{~m}, 2 \mathrm{H}), 1.43$ (sextet, $J=7.6 \mathrm{~Hz}, 2 \mathrm{H}$ ), 1.40 (sextet, $J=7.6 \mathrm{~Hz}, 2 \mathrm{H}), 1.01(\mathrm{t}, J=7.6 \mathrm{~Hz}, 3 \mathrm{H}), 0.99(\mathrm{t}, J=7.6 \mathrm{~Hz}, 3 \mathrm{H}), 0.88(\mathrm{~s}, 9 \mathrm{H}) .{ }^{13} \mathrm{C} \mathrm{NMR}$ $\left(100 \mathrm{MHz}_{,} \mathrm{CDCl}_{3}\right): \delta 162.5,144.5,128.8,120.2\left(\mathrm{q}, J_{\mathrm{C}-\mathrm{F}}=318.9 \mathrm{~Hz}\right), 59.4,54.2,51.1,46.7,42.0,33.4,31.3$, 31.0, 26.5, 25.1, 24.1, 22.6, 19.7, 19.6, 13.5, 13.4. ${ }^{19} \mathrm{~F} \mathrm{NMR}\left(376 \mathrm{MHz}, \mathrm{CDCl}_{3}\right): \delta-79.2$. LRMS (ESI) $\mathrm{m} / z$ (rel int): (pos) $378\left(\left[\mathrm{C} 21 \mathrm{H} 40 \mathrm{~N}_{50}\right]^{+}, 100\right), 383\left(\left[\mathrm{C} 21 \mathrm{H} 40 \mathrm{~N} 5 \mathrm{O}+\mathrm{Na}-\mathrm{H}_{2} \mathrm{O}\right]^{+}, 1\right) . \mathrm{HRMS}(\mathrm{ESI}) \mathrm{m} / \mathrm{z}$ calcd for $\mathrm{C} 21 \mathrm{H} 40 \mathrm{~N}^{2} \mathrm{O}^{+}$378.3227, found 378.3237 .

(S)-4-[4-(4-Isopropyl-2-oxoimidazolidin-1-yl)butyl]-1,3-dibutyl-1H-1,2,3-triazolium

bis(trifluoromethanesulfonyl)imide (2c). 2c was synthesized according to the procedure described for the synthesis of 2a. 16c $(169 \mathrm{mg}, 343 \mu \mathrm{mol})$ and $\mathrm{LiNTf}_{2}(98.6 \mathrm{mg}, 343 \mu \mathrm{mol})$ were used. Reaction time: 24 h. Yield: $197 \mathrm{mg}, 89 \%$. Colorless oil. TLC: $\mathrm{R}_{f} 0.26\left(10: 1 \mathrm{CH}_{2} \mathrm{Cl}_{2} / \mathrm{MeOH}\right) \cdot[\alpha]_{\mathrm{D}}^{20.0}=-29.7\left(\mathrm{c} 1.0, \mathrm{CHCl}_{3}\right)$. ${ }^{1} \mathrm{H} \mathrm{NMR}\left(400 \mathrm{MHz} \mathrm{CDCl}_{3}\right): \delta 8.44(\mathrm{~s}, 1 \mathrm{H}), 4.55(\mathrm{t}, J=7.2 \mathrm{~Hz}, 2 \mathrm{H}), 4.45(\mathrm{t}, J=7.2 \mathrm{~Hz}, 2 \mathrm{H}), 3.56(\mathrm{t}, J=8.8$ $\mathrm{Hz}, 1 \mathrm{H}), 3.46(\mathrm{dt}, J=8.8,7.2 \mathrm{~Hz}, 1 \mathrm{H}), 3.37(\mathrm{dt}, J=14.4,7.2 \mathrm{~Hz}, 1 \mathrm{H}), 3.18(\mathrm{dt}, J=14.4,6.4 \mathrm{~Hz}, 1 \mathrm{H})$, $3.12(\mathrm{dd}, J=8.8,7.6 \mathrm{~Hz}, 1 \mathrm{H}), 2.92(\mathrm{~m}, 2 \mathrm{H}), 1.99$ (quintet, $J=7.2 \mathrm{~Hz}, 2 \mathrm{H}$ ), 1.95 (quintet, $J=7.2 \mathrm{~Hz}$, 2H), $1.80(\mathrm{~m}, 2 \mathrm{H}), 1.73-1.65(\mathrm{~m}, 3 \mathrm{H}), 1.43$ (sextet, $J=7.2 \mathrm{~Hz}, 2 \mathrm{H}), 1.40$ (sextet, $J=7.2 \mathrm{~Hz}, 2 \mathrm{H}), 1.01(\mathrm{t}$, $J=7.2 \mathrm{~Hz}, 3 \mathrm{H}), 0.99(\mathrm{t}, J=7.2 \mathrm{~Hz}, 3 \mathrm{H}), 0.92(\mathrm{~d}, J=6.8 \mathrm{~Hz}, 3 \mathrm{H}), 0.89(\mathrm{~d}, J=6.8 \mathrm{~Hz}, 3 \mathrm{H}) .{ }^{13} \mathrm{C}$ NMR $(100$ $\mathrm{MHz}, \mathrm{CDCl} 3): \delta 162.3,144.3,128.5,119.8\left(\mathrm{q}, \mathrm{J}_{\mathrm{C}-\mathrm{F}}=319.6 \mathrm{~Hz}\right), 56.0,53.9,50.9,48.8,41.7,33.0,31.1,30.8$, 26.3, 24.0, 22.3, 19.5, 19.4, 18.2, 17.7, 13.3, 13.2. ${ }^{19} \mathrm{~F} \mathrm{NMR}\left(376 \mathrm{MHz}, \mathrm{CDCl}_{3}\right): \delta-79.2$. LRMS (ESI) $\mathrm{m} / z$ (rel int): (pos) $378\left(\left[\mathrm{C} 20 \mathrm{H} 38 \mathrm{~N}^{2} \mathrm{O}\right]^{+}, 100\right), 346\left(\left[\mathrm{C} 20 \mathrm{H} 38 \mathrm{~N} 5 \mathrm{O}-\mathrm{H}_{2} \mathrm{O}\right]^{+}, 1\right)$. HRMS (ESI) m/z calcd for $\mathrm{C} 20 \mathrm{H} 38 \mathrm{~N}^{2} \mathrm{O}^{+} 364.3071$, found 364.3066 .

\subsection{Representative Procedure for Ionic Liquid-Supported Asymmetric Benzylation}

In a $1 \mathrm{~mL}$ roundbottom flask, benzylimidazolidinone ionic liquid 2a $(69.3 \mathrm{mg}, 100 \mu \mathrm{mol})$ was dissolved in anhydrous THF $(0.37 \mathrm{~mL})$ at $\mathrm{rt}$, and $n$-butyllithium solution $(44 \mu \mathrm{L}, 110 \mu \mathrm{mol}, 2.5 \mathrm{M}$ in hexanes) was added at $-78{ }^{\circ} \mathrm{C}$. The reaction mixture was then stirred for 20 min and propionyl chloride $(11 \mu \mathrm{L}, 120 \mu \mathrm{mol})$ was added dropwise. After stirring at $-78^{\circ} \mathrm{C}$ for $6 \mathrm{~h}$, the reaction mixture was warmed to rt, quenched with saturated aqueous $\mathrm{NH}_{4} \mathrm{Cl}(1 \mathrm{~mL})$, and diluted with $\mathrm{CH}_{2} \mathrm{Cl}_{2}(5 \mathrm{~mL})$. The mixture was basified with $10 \%$ aqueous $\mathrm{NaOH}(3 \mathrm{~mL})$. The aqueous layer was separated, and extracted with $\mathrm{CH}_{2} \mathrm{Cl}_{2}(2 \times 8 \mathrm{~mL})$. The combined organic layers were washed with brine $(8 \mathrm{~mL}), \mathrm{dried}$ over anhydrous $\mathrm{Na}_{2} \mathrm{SO}_{4}$, filtered, and concentrated in vacuo at $60{ }^{\circ} \mathrm{C}$ for $14 \mathrm{~h}$. The colorless liquid 17 a was used directly in the next step without further purification. In a $1 \mathrm{~mL}$ roundbottom flask, imidazolidinone 17 a was dissolved in anhydrous THF $(0.32 \mathrm{~mL})$ at rt. The reaction mixture was cooled 
to $-78^{\circ} \mathrm{C}$ and NaHMDS solution $(180 \mu \mathrm{L}, 180 \mu \mathrm{mol}, 1 \mathrm{M}$ in THF) was added. The mixture was then stirred for $1 \mathrm{~h}$, and a solution of 3-chlorobenzyl bromide $(26.2 \mu \mathrm{L}, 199 \mu \mathrm{mol})$ in THF $(0.08 \mathrm{~mL})$ was added dropwise, warmed to $0{ }^{\circ} \mathrm{C}$, and stirred for $7 \mathrm{~h}$. Upon completion of the reaction, the reaction mixture was quenched with saturated aqueous $\mathrm{NH}_{4} \mathrm{Cl}(10 \mathrm{~mL})$, warmed to rt, and extracted with EtOAc $(3 \times 20 \mathrm{~mL})$. The combined organic layers were washed with brine $(30 \mathrm{~mL})$ and dried over anhydrous $\mathrm{MgSO}_{4}$, filtered, and concentrated by rotary evaporation. The colorless liquid 18a was used directly in the next step without further purification. In a screw cap vial, benzylated 18a was dissolved in a mixture of anhydrous 1,4-dioxane $(0.30 \mathrm{~mL})$ and $2 \mathrm{~N} \mathrm{NaOH}(0.30 \mathrm{~mL})$ at $\mathrm{rt}$ and the reaction mixture was stirred at $80^{\circ} \mathrm{C}$ for $3.5 \mathrm{~h}$. Upon completion of the reaction, the reaction mixture was cooled to $0{ }^{\circ} \mathrm{C}$ and extracted with $\mathrm{CH}_{2} \mathrm{Cl}_{2}(3 \times 4 \mathrm{~mL})$. The aqueous layer was acidified with $10 \% \mathrm{HCl}$ (5 drops) to $\mathrm{pH} 2$ and extracted with EtOAc $(3 \times 4 \mathrm{~mL})$. The combined $\mathrm{CH}_{2} \mathrm{Cl}_{2}$ layers were dried over anhydrous $\mathrm{Na}_{2} \mathrm{SO}_{4}$, filtered, and concentrated in vacuo to give crude chiral IL 2a. The combined EtOAc layers were dried over anhydrous $\mathrm{Na}_{2} \mathrm{SO}_{4}$, filtered, and concentrated in vacuo to yield analytically pure acid (-)-15 as a colorless liquid (18.0 $\mathrm{mg}, 91 \%)$. The recovered ionic liquid $\mathbf{2 a}(66.7 \mathrm{mg})$ was dried in vacuo and reuse for the next cycle. (-)-15: TLC: Rf $0.20\left(30: 1 \mathrm{CH}_{2} \mathrm{Cl}_{2} / \mathrm{MeOH}\right) \cdot[\alpha]_{\mathrm{D}}^{20.0}=-28.5$ (c 1.0, acetone). ${ }^{1} \mathrm{H}$ NMR $\left(400 \mathrm{MHz}, \mathrm{CDCl}_{3}\right): \delta 7.24-7.18(\mathrm{~m}, 3 \mathrm{H}), 7.07(\mathrm{dt}, J=14.4,1.6 \mathrm{~Hz}, 1 \mathrm{H}), 3.05(\mathrm{dd}, J=13.6,6.8$ $\mathrm{Hz}, 1 \mathrm{H}), 2.78$ (sextet, $J=7.2 \mathrm{~Hz}, 1 \mathrm{H}), 2.67(\mathrm{dd}, J=13.6,7.6 \mathrm{~Hz}, 2 \mathrm{H}), 1.20(\mathrm{~d}, J=7.2 \mathrm{~Hz}, 3 \mathrm{H}) .{ }^{13} \mathrm{C}$ NMR $\left(100 \mathrm{MHz}, \mathrm{CDCl}_{3}\right): \delta 181.9,141.1,134.2,129.7,129.1,127.2,126.7,41.0,38.9,16.5$. HRMS (ESI) m/z calcd

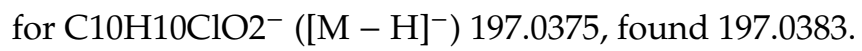

\subsection{Synthesis of N-Phenyl (R)-3-(3-chlorophenyl)-2-methylpropionamide ((R)-S3) for HPLC Analysis}

In a $1 \mathrm{~mL}$ roundbottom flask, acid $(R)-(-)-15(16.0 \mathrm{mg}, 80.5 \mu \mathrm{mol}), \mathrm{DCC}(18.3 \mathrm{mg}, 88.6 \mu \mathrm{mol})$, and DMAP $(0.7 \mathrm{mg}, 5.6 \mu \mathrm{mol})$ were dissolved in anhydrous THF $(0.13 \mathrm{~mL})$ at $\mathrm{rt}$. After aniline $(8.2 \mu \mathrm{L}, 88.6$ $\mu \mathrm{mol}$ ) was added, the mixture was stirred for $2 \mathrm{~h}$ at $\mathrm{rt}$. Upon completion of the reaction, the reaction mixture was filtered through a plug of Celite. The filtrate was diluted with $\mathrm{Et}_{2} \mathrm{O}(5 \mathrm{~mL})$ and washed with $1 \mathrm{~N} \mathrm{HCl}(2 \mathrm{~mL})$. The organic layer was dried over anhydrous $\mathrm{Na}_{2} \mathrm{SO}_{4}$, filtered, and concentrated in vacuo. The purification by column chromatography on silica gel (9:1 hexanes/EtOAc) afforded a desired $(R)-S 3$ (9.5 mg, 43\%, 94\% ee) as a white solid. TLC: Rf 0.16 (9:1 hexanes/EtOAc). ${ }^{1} \mathrm{H}$ NMR (400 $\left.\mathrm{MHz}, \mathrm{CDCl}_{3}\right): \delta 7.38(\mathrm{~d}, J=8.0 \mathrm{~Hz}, 2 \mathrm{H}), 7.30(\mathrm{~m}, 2 \mathrm{H}), 7.22-7.19(\mathrm{~m}, 3 \mathrm{H}), 7.10(\mathrm{~m}, 2 \mathrm{H}), 6.85(\mathrm{br} \mathrm{s}, 1 \mathrm{H})$, $3.05(\mathrm{dd}, J=13.6,8.4 \mathrm{~Hz}, 1 \mathrm{H}), 2.73(\mathrm{dd}, J=13.6,7.2 \mathrm{~Hz}, 1 \mathrm{H}), 2.56($ sextet, $J=6.8 \mathrm{~Hz}, 1 \mathrm{H}), 1.29(\mathrm{~d}, J=6.8$ $\mathrm{Hz}, 3 \mathrm{H}) .{ }^{13} \mathrm{C}$ NMR $\left(100 \mathrm{MHz}, \mathrm{CDCl}_{3}\right): \delta 173.4,141.7,137.5,134.3,129.8,129.0,128.9,127.3,126.7,124.4$, 120.1, 44.7, 40.1, 17.9. HRMS (ESI) m/z calcd for C16H17ClNO ${ }^{+}\left([\mathrm{M}+\mathrm{H}]^{+}\right)$274.0993, found 274.1002.

\section{Conclusions}

The ionic liquid-supported imidazolidinone chiral auxiliaries were developed. The ionic liquid-supported chiral imidazolidinone auxiliaries $\mathbf{1 a - c}$ and $\mathbf{2 a - c}$ were rationally designed and synthesized in 9 steps from commercially available bromobutane. Asymmetric alkylation-cleavage reactions were performed that produced (-)-3-(3-chlorophenyl)-2-methylpropionic acid (15) with high yield and high enantioselectivity. In this study, the problems associated with heterogeneous reactions, such as the difficulty with reaction monitoring, moderate stereoselectivity, nonlinear kinetic behavior, diffusion-limited reactivity, unequal distribution of reagents, low reactivity, extended reaction times, and reagent leaching could be resolved. It is believed that the ionic liquid-supported chiral imidazolidinone auxiliaries could also be widely used for other asymmetric reactions.

Supplementary Materials: The following are available online: Scheme S1 and copies of ${ }^{1} \mathrm{H}$ and ${ }^{13} \mathrm{C}$ spectra of all new compounds synthesized in this work.

Author Contributions: Y.J. synthesized IL-supported imidazolidinones and conducted the application reactions; Y.P. performed comparison experiments using Evans' oxazolidinone; Y.J. and J.-S.R. wrote the manuscript. Y.P. and J.-S.R. revised the manuscript; Conceptualization, J.-S.R.; methodology, Y.J.; validation, Y.J. and Y.P.; investigation, Y.J. and Y.P.; resources, J.-S.R.; data curation, Y.J. and Y.P.; writing-original draft preparation, Y.J. 
and J.-S.R.; writing—review and editing, Y.J. and J.-S.R.; supervision, J.-S.R.; project administration, J.-S.R.; funding acquisition, J.-S.R.

Funding: This research was supported by National Research Foundation (NRF) funded by the Korean government (MSIT), the Bio \& Medical Technology Development Program (NRF-2017M3A9F5029066) and MRC Program (2018R1A5A2025286).

Conflicts of Interest: The authors declare no conflicts of interest.

\section{References and Notes}

1. Evans, D.A.; Takacs, J.M. Enantioselective alkylation of chiral enolates. Tetrahedron Lett. 1980, 21, 4233-4236. [CrossRef]

2. Heravi, M.M.; Zadsirjan, V. Oxazolidinones as chiral auxiliaries in asymmetric aldol reactions applied to total synthesis. Tetrahedron Asymmetry 2013, 24, 1149-1188. [CrossRef]

3. Evans, D.A.; Bartroli, J.; Shih, T.L. Enantioselective aldol condensations. 2. Erythro-selective chiral aldol condensations via boron enolates. J. Am. Chem. Soc. 1981, 103, 2127-2129. [CrossRef]

4. Heravi, M.M.; Zadsirjan, V.; Farajpour, B. Applications of oxazolidinones as chiral auxiliaries in the asymmetric alkylation reaction applied to total synthesis. RSC Adv. 2016, 6, 30498-30551. [CrossRef]

5. Evans, D.A.; Ennis, M.D.; Mathre, D.J. Asymmetric alkylation reactions of chiral imide enolates. A practical approach to the enantioselective synthesis of $\alpha$-substituted carboxylic acid derivatives. J. Am. Chem. Soc. 1982, 104, 1737-1739. [CrossRef]

6. Evans, D.A.; Chapman, K.T.; Bisaha, J. New asymmetric Diels-Alder cycloaddition reactions. Chiral $\alpha, \beta$-unsaturated carboximides as practical chiral acrylate and crotonate dienophile synthons. J. Am. Chem. Soc. 1984, 106, 4261-4263. [CrossRef]

7. Phoon, C.W.; Abell, C. Solid phase aldol and conjugate addition reactions using Evans' oxazolidinone chiral auxiliary. Tetrahedron Lett. 1998, 39, 2655-2658. [CrossRef]

8. For recent reviews see: Chung, C.W.Y.; Toy, P.H. Chiral auxiliaries in polymer-supported organic synthesis. Tetrahedron Asymmetry 2004, 15, 387-399.

9. Desimoni, G.; Faita, G.; Galbiati, A.; Pasini, D.; Quadrelli, P.; Rancati, F. A soluble polymer-bound Evans' chiral auxiliary: Synthesis, characterization and use in cycloaddition reactions. Tetrahedron Asymmetry 2002, 13, 333-337. [CrossRef]

10. Faita, G.; Paio, A.; Quadrelli, P.; Rancati, F.; Seneci, P. Solid supported chiral auxiliaries in asymmetric synthesis. Part 2: Catalysis of 1,3-dipolar cycloadditions by Mg(II) cation. Tetrahedron 2001, 57, 8313-8322. [CrossRef]

11. Burgess, K.; Lim, D. Resin type can have important effects on solid phase asymmetricalkylation reactions. Chem. Commun. 1997, 785-786. [CrossRef]

12. Hutchison, P.C.; Heightman, T.D.; Procter, D.J. Application of a recyclable pseudoephedrine resin in asymmetric alkylations on solid phase. J. Org. Chem. 2004, 69, 790-801. [CrossRef] [PubMed]

13. Bew, S.P.; Bull, S.D.; Davies, S.G.; Savory, E.D.; Watkin, D.J. Rearrangements and racemisation during the synthesis of 1-serine derived oxazolidin-2-ones. Tetrahedron 2002, 58, 9387-9401. [CrossRef]

14. Bew, S.P.; Bull, S.D.; Davies, S.G. Polymer supported oxazolidin-2-ones derived from l-serine-A cautionary tale. Tetrahedron Lett. 2000, 41, 7577-7581. [CrossRef]

15. Shi, R.R.; Wang, F.; Yan, B. Site-site isolation and site-site interaction-Two sides of the same coin. Int. J. Pept. Res. Ther. 2007, 13, 213-219. [CrossRef]

16. Dickerson, T.J.; Reed, N.N.; Janda, K.D. Soluble polymers as scaffolds for recoverable catalysts and reagents. Chem. Rev. 2002, 102, 3325-3344. [CrossRef]

17. Mutter, M. Soluble polymers in organic synthesis: I. Preparation of polymer reagents using polyethylene glycol with terminal amino groups as polymeric component. Tetrahedron Lett. 1978, 31, 2839-2842. [CrossRef]

18. Pillai, V.N.R.; Mutter, M.; Bayer, E.; Gatfield, I. New, easily removable poly(ethylene glycol) supports for the liquid-phase method of peptide synthesis. J. Org. Chem. 1980, 45, 5364-5370. [CrossRef]

19. Shemyakin, M.M.; Ovchinnikov, Y.A.; Kiryushkin, A.A.; Kozhevnikova, I.V. Synthesis of peptides in solution on a polymeric support I. Synthesis of glycylglycyl-L-leucylglycine. Tetrahedron Lett. 1965, 27, $2323-2327$. [CrossRef] 
20. Bergbreiter, D.E. Using soluble polymers to recover catalysts and ligands. Chem. Rev. 2002, 102, $3345-3384$. [CrossRef]

21. Han, H.; Wolfe, M.M.; Brenner, S.; Janda, K.D. Liquid-phase combinatorial synthesis. Proc. Natl. Acad. Sci. USA 1995, 92, 6419-6423. [CrossRef]

22. Miao, W.; Chan, T.H. Ionic-liquid-supported synthesis: A novel liquid-phase strategy for organic synthesis. Acc. Chem. Res. 2006, 39, 897-908. [CrossRef]

23. Ni, B.; Headley, A.D. Ionic-liquid-supported (ILS) catalysts for asymmetric organic synthesis. Chem. Eur. J. 2010, 16, 4426-4436. [CrossRef]

24. Jeong, Y.; Ryu, J.-S. Synthesis of 1,3-dialkyl-1,2,3-triazolium ionic liquids and their applications to the Baylis-Hillman reaction. J. Org. Chem. 2010, 75, 4183-4191. [CrossRef]

25. Jeong, Y.; Kim, D.Y.; Choi, Y.; Ryu, J.-S. Intramolecular hydroalkoxylation in Brønsted acidic ionic liquids and its application to the synthesis of ( \pm )-centrolobine. Org. Biomol. Chem. 2011, 9, 374-378. [CrossRef]

26. For recent reviews on the Huisgen 1,3-dipolar cycloaddition reaction see: Meldal, M.; Tornøe, C.W. Cu-Catalyzed Azide-Alkyne Cycloaddition. Chem. Rev. 2008, 108, 2952-3015.

27. Chavda, S.; Coulbeck, E.; Dingjan, M.; Eames, J.; Motevalli, M. Probing the parallel resolution of Mosher's acid using a combination of quasi-enantiomeric oxazolidin-2-ones. Tetrahedron Asymmetry 2008, 19, 1274-1284. [CrossRef]

28. Leisch, H.; Sullivan, B.; Fonovic, B.; Dudding, T.; Hudlicky, T. New options for the reactivity of the Burgess reagent with epoxides in both racemic and chiral auxiliary modes - structural and mechanistic revisions, computational studies, and application to synthesis. Eur. J. Org. Chem. 2009, 2806-2819. [CrossRef]

29. Jindal, R.; Sablok, A. Preparation and applications of room temperature ionic liquids in organic synthesis: A review on recent efforts. Curr. Green Chem. 2015, 2, 135-155. [CrossRef]

30. Evans, D.A.; Britton, T.C.; Ellman, J.A. Contrasteric carboximide hydrolysis with lithium hydroperoxide. Tetrahedron Lett. 1987, 28, 6141-6144. [CrossRef]

31. Evans, D.A.; Ellman, J.A. The total syntheses of the isodityrosine-derived cyclic tripeptides OF4949-III and K-13. Determination of the absolute configuration of K-13. J. Am. Chem. Soc. 1989, 111, 1063-1072. [CrossRef]

Sample Availability: Samples of the compounds are available from the authors.

(C) 2019 by the authors. Licensee MDPI, Basel, Switzerland. This article is an open access article distributed under the terms and conditions of the Creative Commons Attribution (CC BY) license (http://creativecommons.org/licenses/by/4.0/). 